\title{
Comparación de la flora exótica vascular en sistemas de islas continentales: Cerdeña (Italia) y Baleares (España)
}

\author{
por \\ Lina Podda ${ }^{1}$, Pere Fraga i Arguimbau ${ }^{2}$, Olga Mayoral García-Berlanga ${ }^{3}$, Francesco Mascia ${ }^{1} \&$ Gianluigi Bacchetta $^{1}$ \\ ${ }^{1}$ Centro Conservazione Biodiversità (CCB), Dipartimento di Scienze Botaniche, Università degli Studi di Cagliari, \\ v.le S. Ignazio da Laconi 13, 09123 Cagliari, Italy. info@ccb-sardegna.it \\ ${ }^{2}$ Departament Econ. i Medi Amb., Consell Insular de Menorca, Menorca, Illes Balears, España. pfa.life@cime.es \\ ${ }^{3}$ Instituto de Investigación para la Gestión Integrada de Zonas Costeras (IGIC), Departamento de Ecosistemas Agroforestales, \\ Escuela Politécnica Superior de Gandía, Universidad Politécnica de Valencia, Avda. Paraninfo 1, 46730, Grao de Gandía, \\ Gandía, Valencia, España; Departamento de Botànica, Facultad de Ciencias Biológicas, Universidad de Valencia, \\ Dr. Moliner 50, 46100 Burjassot, Valencia, España. olmagar@eaf.upv.es
}

\section{Resumen}

Podda, L., Fraga i Arguimbau, P., Mayoral García-Berlanga, O., Mascia, F. \& Bacchetta, G. 2010. Comparación de la flora exótica vascular en sistemas de islas continentales: Cerdeña (Italia) y Baleares (España). Anales Jard. Bot. Madrid 67(2): 157-176.

Se presenta un estudio comparativo de la flora vascular exótica de Cerdeña y de las Baleares, dos sistemas insulares pertenecientes a la subregión biogeográfica Mediterránea Occidental. En Cerdeña se han contabilizado 531 táxones exóticos (18,8\% del total de su flora), mientras que en las Baleares 360 (19\%), siendo 10 citas nuevas para Cerdeña (3 de las cuales para Italia) y 29 para Baleares. La flora exótica de Cerdeña está incluida en 99 familias, y Fabaceae es la más rica (49 táxones), seguida por Poaceae (33) y Asteraceae (31), frente a 90 familias para las Baleares, con predominio de Fabaceae (32), Asteraceae (31) y Poaceae (27). Se han encontrado diferencias respecto a los tipos biológicos, con un predominio de los fanerófitos en Cerdeña y de los terófitos en las Baleares. Un análisis detallado muestra que buena parte de estos táxones (246) son compartidos por ambos territorios, así como una dominancia de los neófitos frente a los arqueófitos. Respecto al origen geográfico, ambos territorios presentan una preeminencia del elemento americano sobre el mediterráneo. En referencia a las vías de introducción, la mayor parte de los táxones ha sido introducida por parte del hombre de forma intencionada (76\% SA, 77\% BL) en particular para uso ornamental (43\% SA, 45\% BL). Los hábitats más afectados son los seminaturales, agrícolas y sinantrópicos en ambos territorios, aunque atendiendo a la flora invasora, son los litorales los más sensibles en Cerdeña y los humedales en Baleares. Una parte del trabajo aborda las causas de la fragilidad y baja resiliencia de los diferentes hábitats.

Palabras clave: biogeografía, conservación, especies exóticas, invasiones, Mediterráneo.

\begin{abstract}
Podda, L., Fraga i Arguimbau, P., Mayoral García-Berlanga, O., Mascia, F. \& Bacchetta, G. 2010. Comparison of the vascular exotic flora in continental islands: Sardinia (Italy) and Balearic Islands (Spain). Anales Jard. Bot. Madrid 67(2): 157-176 (in Spanish).

This paper provides a comparison of the vascular exotic flora of Sardinia and that of the Balearic Islands, both territories belonging to the Western Mediterranean biogeographic subregion. The study has recorded 531 exotic taxa in Sardinia (18.8\% of the total flora) while 360 (19\%) in the Balearic Islands; 10 are new to Sardinia (3 of which for Italy) and 29 to the Balearic Islands. The alien flora of Sardinia is included in 99 families; Fabaceae is the richest (49 taxa), followed by Poaceae (33) and Asteraceae (31) while in the Balearic Islands in 90 families, with a predominance of Fabaceae (32), Asteraceae (31) and Poaceae (27). The comparison of the biological spectra reveals that in Sardinia phanerophytes are the most represented in Sardinia and therophytes in the Balearic Islands. A detailed analysis shows that most of the exotic taxa (246) are shared by both territories with a clear dominance of neophytes rather than archaeophytes. A study of the geographical origin shows supremacy of the American element over the Mediterranean. The majority of introduced exotic taxa are a result of intentional human introductions (76\% SA, 77\% BL), mainly for ornamental use (43\% SA, $45 \% \mathrm{BL})$. The most occupied habitats are the semi-natural, agricultural and synanthropic for both territories, but attending to invasive plants, coastal habitats in Sardinia and wetlands in the Balearic Islands are the most sensitive. A part of the work deals with the causes of fragility and low resilience of the different habitats.
\end{abstract}

Keywords: alien species, biogeography, conservation, invasions, Mediterranean. 


\section{Introducción}

Los estudios comparativos relativos a la flora exótica, desarrollados en territorios próximos y relacionados desde el punto de vista biogeográfico, adquieren particular importancia en islas, tanto oceánicas como continentales (Crawley \& al., 1996; Fritts \& Rodda, 1998; Cox, 1999; Sherley, 2000; Clout \& Veitch, 2002; Hernández \& al., 2002; Daehler \& al., 2004; Kueffer, 2006).

En los sistemas insulares mediterráneos, la alta tasa de endemicidad, de especies en peligro de extinción y de hábitats sensibles, unido al milenario impacto de las actividades humanas, las convierten en zonas prioritarias para la conservación (Heywood, 1989; Kunkel, 1993; Mayer, 1995; Natali \& Jeanmonod, 1996; Jeanmonod, 1998; Le Neindre, 2002; Sanz Elorza \& al., 2004; Blondel \& Médail, 2009). Por otra parte, las islas y las floras propias de los territorios insulares, son altamente vulnerables frente a las invasiones biológicas (Vitousek, 1987; Lodge, 1993; Suehs \& al., 2001, 2004; Moragues \& Rita, 2005; Celesti-Grapow $\&$ al., 2009a; DAISIE, 2009) y demuestran una menor capacidad competitiva respecto a las especies exóticas (Simberloff, 1995, 2000; Lloret \& al., 2004; Pyšek \& Richardson, 2006), lo que conlleva una menor resiliencia de los ecosistemas particularmente evidente y ampliamente demostrada en islas oceánicas (Towns \& Ballantine, 1993; Cronk \& Fuller, 1995; Kitayama \& Mueller-Dombois, 1995; Olesen \& al., 2002; Denslow, 2003; O’Dowd \& al., 2003; Tassin \& al., 2006; Kueffer \& al., 2010). De hecho, es a partir del momento en que se reduce el grado de resiliencia de los ecosistemas naturales y la competitividad por parte de la flora autóctona cuando la vulnerabilidad aumenta, creándose un desequilibrio permanente aprovechado por las especies exóticas y en particular por las que tienen carácter invasor (MuellerDombois, 1981; Loope \& Mueller-Dombois, 1989; Mooney \& Hobbs, 2000; Weber, 2003; Levine \& al., 2004; D'Antonio \& al., 2009). Este fenómeno resulta directamente proporcional a la creciente presión antrópica y al desaforado desarrollo turístico de las últimas décadas en las islas continentales mediterráneas (Debussche \& al., 1999), que producen un fuerte impacto ambiental y socio-económico (Mack \& al., 2000; Reaser \& al., 2007; Gillespie \& al., 2008).

En el Mediterráneo, durante los últimos cinco años, se han realizado multitud de estudios en áreas insulares relativos a especies consideradas invasoras a escala internacional (p. ej., Brundu \& al., 2004; Lloret \& al., 2004; Vilà \& al., 2004, 2006; Hulme \& al., 2008b; Bacchetta \& al., 2010); sin embargo, son esca- sas las contribuciones que consideran el conjunto de la flora exótica de un territorio, comparándola con la flora autóctona y con las floras, tanto exóticas como autóctonas, de áreas pertenecientes a la misma región biogeográfica mediterránea.

Para las islas mediterráneas, Lloret \& al. (2005) aportan una serie de atributos de aquellas especies de mayor éxito invasor, comparando datos de las islas de Córcega, Creta, Mallorca, Malta y Cerdeña que permiten explicar la abundancia de un número importante de táxones naturalizados. Respecto a la isla de Sicilia y los territorios insulares próximos, Giardina \& al. (2007) presentan un catálogo de la flora vascular especificando todos los táxones exóticos. Recientemente, Bacchetta \& al. (2009b) realizan un catálogo florístico de la flora exótica de Cerdeña y comparan la misma con el conjunto de la flora vascular de la isla. De la misma forma, Moragues \& Rita (2005) elaboran un listado de toda la flora exótica de las Baleares, Dal Cin D'Agata \& al. (2009) presentan un primer inventario de la flora exótica de Creta, mientras Bazos \& al. (2009) tratan de analizar desde el punto de vista corológico el conjunto de la flora vascular exótica de Grecia, considerando también los territorios insulares, pero sin hacer comparaciones.

A pesar de todo esto, diversos estudios apuntan la importancia de comparar las floras invasoras de áreas con características climáticas análogas, con patrones estacionales similares en cuanto a temperatura, distribución y cantidad de precipitaciones a lo largo del año (Di Castri, 1991; Le Houérou, 1991; Lonsdale, 1999; Pauchard \& al., 2004). Estas comparaciones son más coincidentes dentro de una misma región biogeográfica, en nuestro caso, la región mediterránea. La comparación dentro de una misma región biogeográfica permite asimismo incrementar el conocimiento sobre el comportamiento de las exóticas para ayudar en el campo de la gestión y prevención, así como a la hora de elaborar políticas conjuntas. Sin embargo, este tipo de comparaciones conlleva dificultades debido a la heterogeneidad de los datos, en ocasiones incompletos o significativamente diferentes en cuanto al formato y metodología empleados.

Con el presente trabajo, a partir de los estudios anteriormente citados y de la base de datos DAISIE (2010), se pretende aportar un análisis comparativo entre la flora vascular exótica de Cerdeña y Baleares, haciendo también una comparación con las floras generales de ambos sistemas insulares, proporcionando datos inéditos respecto a táxones exóticos que constituyen nuevas citas para Cerdeña, Baleares o sus respectivos estados. Asimismo se analizan y comparan las vías de introducción de la flora exótica y se identi- 
fican los hábitats más sensibles a las invasiones, discutiendo las causas de la fragilidad y baja resiliencia de los mismos en ambos territorios.

La finalidad última de este trabajo es ofrecer suficientes datos para poder elaborar en un futuro próximo listados de prioridad de actuación (Blasi \& al., 2008; Randall \& al., 2008) y proyectos de gestión, encaminados a la mitigación de los efectos de las invasiones biológicas y al establecimiento de una red de monitoreo y prevención en el ámbito interinsular.

\section{Área de estudio}

Cerdeña se localiza en la parte central del Mediterráneo occidental (Fig. 1), entre los 38 51'52" (cabo Teulada) y los $41^{\circ} 15^{\prime} 42^{\prime \prime}$ (cabo Falcone) de latitud norte y los $8^{\circ} 8^{\prime} 10^{\prime \prime}$ (cabo de la Argentiera) y los $9^{\circ} 50$ '08" (cabo Comino) de longitud este a partir de Greenwich.

El archipiélago Balear (Fig. 1) se encuentra en una localización geográfica que se sitúa entre los $40^{\circ} 05^{\prime} 44^{\prime}$ de la isla de Sanitja (o des Porros), en Me- norca y $38^{\circ} 38^{\prime} 32^{\prime \prime}$ de latitud norte del cabo de Berbería (Barbaria), en Formentera, y los 4'19'29" de la punta de s'Esperó, en Menorca y 109'37" de longitud este de la isla Bleda Plana, en Ibiza.

Entre los dos puntos más próximos de Cerdeña (cabo Caccia) y Baleares (punta de s'Esperó) hay una distancia de $332 \mathrm{~km}$.

Cerdeña, incluidas también las 300 islas e islotes que la rodean, tiene una superficie de $24089 \mathrm{~km}^{2}$ y representa la segunda isla más grande del Mediterráneo, después de Sicilia $\left(25708 \mathrm{~km}^{2}\right)$. No posee una orografía con montañas muy elevadas, con sólo el 13,6\% del territorio por encima de los $600 \mathrm{~m}$, alcanzando una altitud máxima de $1834 \mathrm{~m}$ en la punta La Marmora (macizo Gennargentu). El perímetro costero de Cerdeña y las islas circumsardas es de unos $1995 \mathrm{~km}$ y está dominado por costas altas y rocosas y secundariamente por costas bajas y sistemas dunares.

El archipiélago de las Islas Baleares tiene una superficie total de $4992 \mathrm{~km}^{2}$ y está formado por cinco islas principales, que de mayor a menor superficie son:

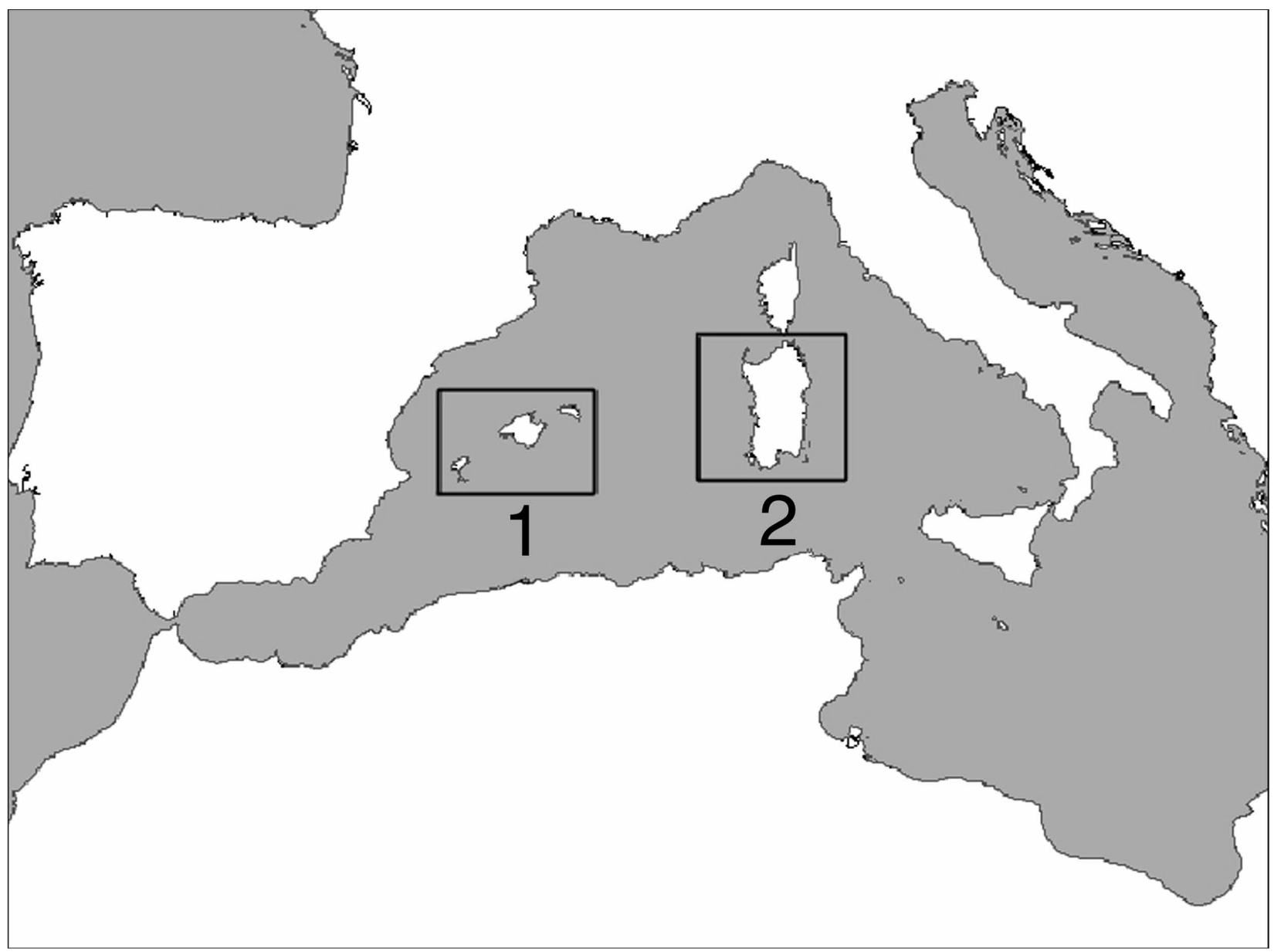

Fig. 1. Localización del área de estudio en la cuenca mediterránea occidental: 1, Baleares; 2, Cerdeña. 
Mallorca, Menorca, Ibiza (Eivissa), Formentera y Cabrera, y númerosos islotes de menor entidad. El perímetro de sus costas tiene una longitud total de $1428 \mathrm{~km}$. En cuanto a la orografía, el relieve es en general bajo. Las alturas más importantes se sitúan en la parte septentrional de la isla de Mallorca (serra de Tramuntana) con una altura máxima de $1445 \mathrm{~m}$, mientras que las otras islas no superan los $500 \mathrm{~m}$ (475 m en Ibiza y $365 \mathrm{~m}$ en Menorca). Desde el punto de vista geográfico, las Baleares se dividen en dos subarchipiélagos: las Gimnesias (Mallorca, Menorca y Cabrera) y las Pitiusas (Ibiza y Formentera).

Cerdeña y las Baleares han atravesado las mismas vicisitudes paleogeográficas; de acuerdo con las reconstrucciones tectónicas (Álvarez, 1972; Rosenbaum \& al., 2002) y paleogeográficas actuales (Mansion \& al., 2008), la microplaca cabilio-baleárica y la corsosardo-calabresa, se separaron de la Península Ibérica hace entre 30 y 28 millones de años. Ambas placas estuvieron sometidas a un fenómeno de rotación y translación que produjo la separación del archipiélago Balear de Cabilia, su establecimiento (hace alrededor de 21 m.a.) en la posición actual, la colisión de la microplaca corso-sarda-calabresa con la apuliana y la separación, primero de Córcega (16 m.a.) y luego de Cerdeña ( 5 m.a.) hasta la posición actual (Rosenbaum $\&$ al., 2002; Speranza \& al., 2002).

Las dos áreas de estudio presentan muchas similitudes desde el punto de vista climático y bioclimático. Debido a su ubicación geográfica y su condición de insularidad, tienen un clima marcadamente oceánico, con una estación templada y húmeda, que va desde el otoño hasta la primavera y una estación cálida y seca que suele durar todo el verano. En términos bioclimáticos, Cerdeña posee un bioclima mediterráneo pluviestacional oceánico y uno templado oceánico de variante submediterránea. Los termotipos varían desde el termomediterráneo inferior hasta el supramediterráneo superior en relación al bioclima mediterráneo y desde el mesotemplado superior al supratemplado superior para el bioclima templado. Desde el punto de vista ombrotérmico, se distinguen cuatro tipologías: semiárido (sólo en el horizonte superior), seco, subhúmedo y húmedo (Bacchetta $\&$ al., 2009a).

En las Baleares el patrón bioclimático está considerado únicamente como un bioclima mediterráneo pluviestacional oceánico. Los termotipos varían desde el termomediterráneo, predominante en la mayoría de las islas y especialmente en la mitad meridional del archipiélago (Pitiusas y parte meridional de Mallorca), pasando por el mesomediterráneo que se localiza en las regiones montañosas de Mallorca (Serra de Tra- muntana y Serra de Llevant) y la parte interior de Menorca, hasta el supramediterráneo inferior que únicamente se produce en los puntos más altos de la región montañosa de Mallorca (Serra de Tramuntana). Desde el punto de vista ombrotérmico, se distinguen tres tipologías: semiárido (sólo en el horizonte superior), seco y subhúmedo.

Desde una perspectiva biogeográfica, las zonas de estudio pertenecen a la subregión Mediterránea Occidental (Rivas-Martínez, 2007). En particular, la subprovincia Sarda se enmarca en la provincia SardoCorsa de la superprovincia Italo-Tirrénica (Ladero Álvarez \& al., 1987; Bacchetta \& Pontecorvo, 2005; Bacchetta \& al., 2009a) y la subprovincia Baleárica en la provincia Catalano-Provenzal-Balear (Rivas-Martínez, 2007).

\section{Material y métodos}

Para realizar las comparaciones de la flora exótica de los dos territorios estudiados se han empleado los datos del catálogo de flora exótica de Cerdeña (Bacchetta \& al., 2009b) y de Baleares (Vilà \& Muñoz, 1999; Moragues \& Rita, 2005), complementados a partir de un trabajo realizado durante los últimos 5 años en el que se han llevado a cabo prospecciones de campo, así como revisiones de diversos herbarios (BC, CAG, CAT, FI, Fragense, GAN, MA, NAP, PAL, SASSA, SS, TO, VAL) y bibliográficas. En particular se ha utilizado como referencia bibliográfica más actualizada la base de datos DAISIE (2010). En los listados los táxones han sido ordenados basándose en el esquema terminológico propuesto en el último catálogo de la flora exótica italiana de Celesti-Grapow $\&$ al. (2009b), en el que se consideran los criterios propuestos por Richardson \& al. (2000), elaborados por Pyšek \& al. (2004) y revisados de acuerdo con Rejmánek \& al. (2004), Richardson \& Pyšek (2006) y Ricciardi \& Cohen (2007).

Para el caso concreto de las Islas Baleares, se han considerado éstas como una unidad geográfica, aunque sin duda su tratamiento individualizado proporcionaría información y resultados de mayor interés. La razón de este tratamiento es que actualmente no existen catálogos detallados de flora vascular para cada uno de los territorios insulares que forman este archipiélago. El análisis cuantitativo más reciente corresponde a Rita \& Payeras (2006), que a su vez tiene como documento de referencia Pla \& al. (1992), en el que no se hace una recopilación exhaustiva de la flora exótica. Únicamente en el caso de Menorca se dispone de una actualización reciente (Fraga \& al., 2004) en la que se establece una diferenciación entre flora vascular autóctona y exótica. 
Asimismo se han diferenciado las especies arqueófitas de las neófitas, en función de su introducción anterior o posterior a 1492/1500, respectivamente. Los cálculos y la elaboración de los resultados han sido realizados considerando todas las especies a excepción de las dudosas (D), incluyendo en esta categoría aquellas de las que no existen citas recientes que demuestren su presencia real o respecto a las cuales hay dudas relativas a su origen geográfico.

No han sido consideradas las especies cultivadas, ornamentales o empleadas para reforestaciones, de las que no se tiene constancia de su naturalización, sino sólo aquellas señaladas por los autores como espontaneizadas.

Los tipos o formas biológicas se basan en la clasificación de Raunkiaer (1934), empleando las siglas de Pignatti (1982).

El origen de las especies se basa en el área de procedencia o en la región biogeográfica y sigue los mismos criterios empleados por Moragues \& Rita (2005) y Bacchetta \& al. (2009b).

Los táxones han sido clasificados según la introducción intencionada o casual por parte del hombre atendiendo a las definiciones propuestas por la Convention on Biological Diversity (CBD) (Miller \& al., 2006). Para el análisis de las vías de introducción han sido utilizadas las categorías propuestas en los trabajos de Sanz Elorza \& al. (2004) y Hulme \& al. (2008b).

Para poder elaborar los datos relativos a los hábitats sensibles se han empleado los criterios propuestos por Vilà \& Muñoz (1999) y Bacchetta \& al. (2009b). Los hábitats han sido señalados en función del medio en que se encuentran los táxones con más frecuencia, siguiendo siete categorías: 1) agrícolas-rurales; 2) sinantrópicos; 3 ) litorales (arenosos y rocosos); 4) arbustivos (garrigas y maquias); 5) forestales; 6) riparios; 7) humedales (dulceacuícolas, salobres y salinos).

Para estudiar las diferencias entre la flora exótica de Cerdeña y de Baleares se aplicó el U-test no paramétrico de Mann-Whitney mediante el uso de la versión 15 (Minitab Inc.) de MINITAB $^{\odot}$.

\section{Resultados}

La revisión del catálogo de la flora vascular exótica de la isla de Cerdeña (Bacchetta \& al., 2009b) da como resultado un total de 531 táxones $(59$ de ellos dudosos), de los cuales 10 son nuevos para la isla y 3 de ellos para Italia (Tabla 1). Respecto al total de su flora vascular (Conti \& al., 2005; Conti \& al., 2007), Cerdeña presenta un $18,8 \%$ de exóticas. Si comparamos estos datos con los de las Baleares según la última estimación de la flora vascular (Rita \& Payeras, 2006), la proporción resulta similar: un 19,0\% para un total de 360 entidades taxonómicas (13 dudosas) actualmente reconocidas como exóticas para las Baleares. Respecto a los datos sobre la flora exótica de Moragues \& Rita (2005), se establece un incremento de 40 táxones, 29 de ellos fruto de las prospecciones de campo y nuevos para las Baleares (Tabla 2) y 11 recopilados a partir de fuentes bibliográficas (Rodríguez, 1904; Bolòs \& al., 1970; Pla \& al., 1992; Fraga \& Pallicer, 1998; Fraga \& al., 2000; Fraga \& al., 2001; Fraga \& al., 2002; Fraga \& al., 2004; Fraga \& García, 2004), de los cuales se ha comprobado su persistencia actual en el medio natural, aun siendo citaciones de varias decenas de años de antigüedad, como es el caso de Coronilla glauca o Stipa tenacisima (Rodríguez, 1904; Bolòs \& al., 1970).

La flora exótica de Cerdeña está incluida en 99 familias; Fabaceae es la más rica (49 táxones), seguida por Poaceae (33) y Asteraceae (31). La flora exótica de las Baleares incluye 90 familias, siendo las más representadas Fabaceae (32), Asteraceae (31) y Poaceae (27) (Fig. 2). Aizoaceae (12 en SA y 14 en BL), Cactaceae (10 en SA y 9 en BL) y Agavaceae ( 9 en SA y 6 en BL) sólo poseen táxones exóticos. El test de Mann-Whitney ha mostrado que, en relación con las familias, no existen diferencias significativas entre las medianas de las muestras consideradas $(\mathrm{U}=14$, $p=0,6958)$.

Un total de 246 táxones exóticos de Cerdeña (52\% del total) son comunes con las Baleares y suponen el $71 \%$ de la flora exótica de las Baleares. De estas 246 entidades en común, el 52\% son especies naturalizadas, el 32\% subespontáneas y el 16\% invasoras para Cerdeña; mientras para las Baleares son el $47 \%$ naturalizadas, el 37\% subespontáneas y el 16\% invasoras (Tabla 3). El test de Mann-Whitney ha demostrado que no hay diferencias significativas entre las medianas de las muestras analizadas ( $\mathrm{U}=3, p=1,000)$.

La flora invasora representa el 12\% de la flora exótica de Cerdeña y el 14\% de las Baleares, mientras que si se considera la flora común a ambos territorios, asciende a un 16\% (Tabla 3). Los datos relativos al total de las plantas subespontáneas muestran una mayor proporción de este estatus en Cerdeña (42\%) que en Baleares (34\%), mientras que las naturalizadas están representadas en mayor porcentaje en Baleares (52\%) que en Cerdeña $(46 \%)$, siendo este último el estatus mayoritario en los dos territorios. El análisis de la flora exótica común a ambos territorios arroja datos muy similares a su análisis por separado para el estatus de naturalizada y subespontánea. El test de Mann-Whitney ha confirmado que no existen diferencias significativas entre las medianas de las mues$\operatorname{tras}(\mathrm{U}=3, p=1,000)$. 


\begin{tabular}{|c|c|c|c|c|c|c|c|c|c|c|c|c|c|c|}
\hline 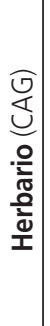 & 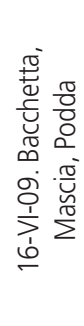 & & & & 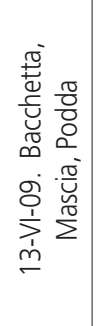 & 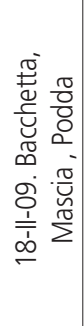 & & 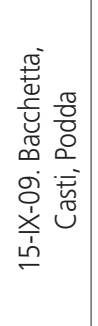 & 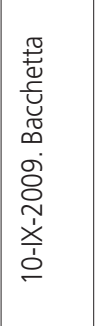 & 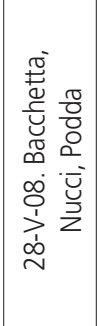 & 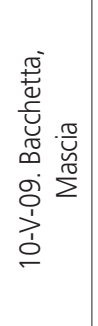 & 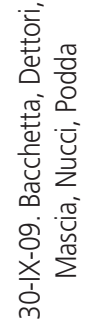 & 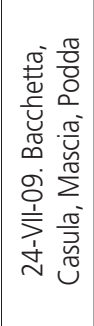 & 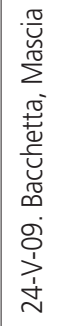 \\
\hline 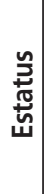 & 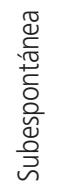 & 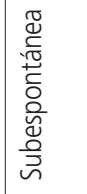 & 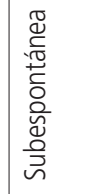 & 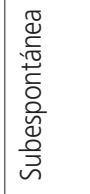 & 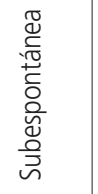 & 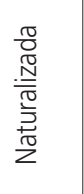 & 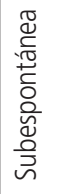 & 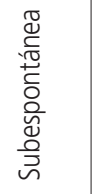 & 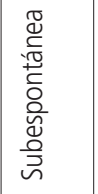 & 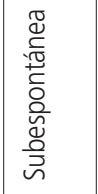 & 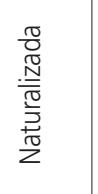 & 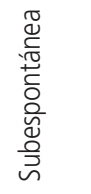 & 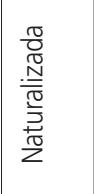 & 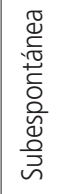 \\
\hline 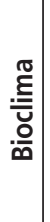 & 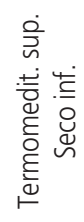 & 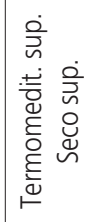 & 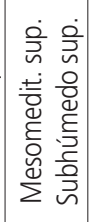 & 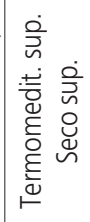 & 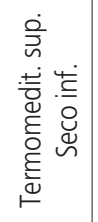 & 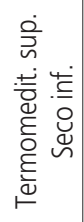 & 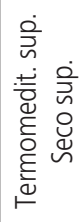 & 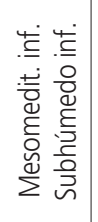 & 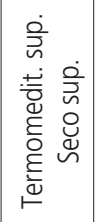 & 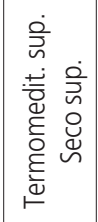 & 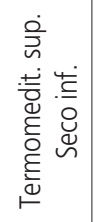 & 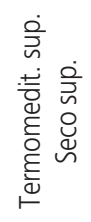 & 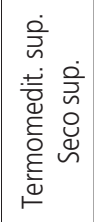 & 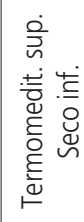 \\
\hline $\begin{array}{l}0 \\
0 \\
\frac{2}{5} \\
\underline{n} \\
n\end{array}$ & 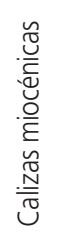 & 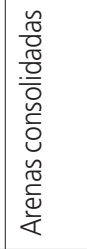 & 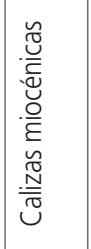 & 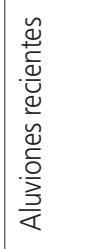 & 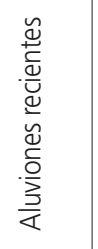 & 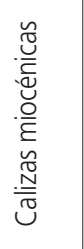 & 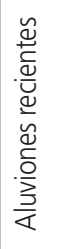 & 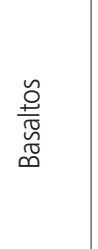 & 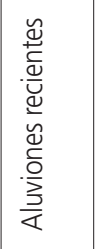 & 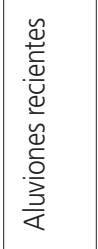 & 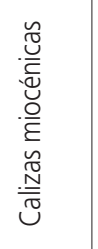 & 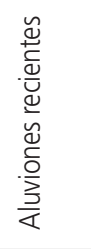 & 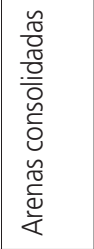 & 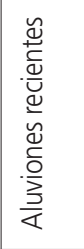 \\
\hline $\begin{array}{c}\hat{\varepsilon} \\
\dot{+}\end{array}$ & N & 0 & $\begin{array}{l}\stackrel{\stackrel{L}{0}}{\circ} \\
\llcorner\end{array}$ & $\bar{\sigma}$ & 0 & 우 & - & $\stackrel{\text { Ð }}{\circ}$ & $\stackrel{\Perp}{-}$ & $\stackrel{m}{\square}$ & $m$ & ్ㅛ & $\nabla$ & 6 \\
\hline 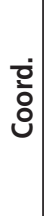 & 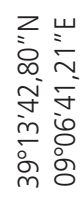 & 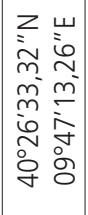 & 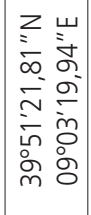 & 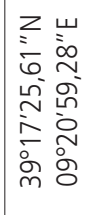 & 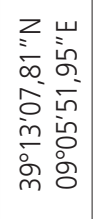 & 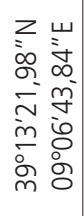 & 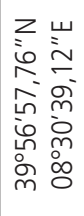 & 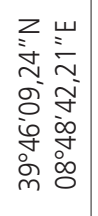 & 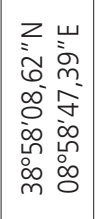 & 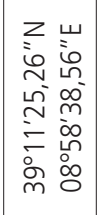 & 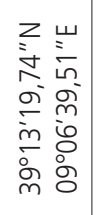 & 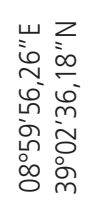 & 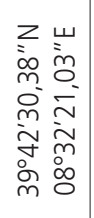 & 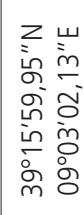 \\
\hline 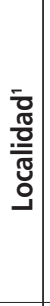 & 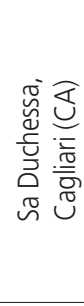 & 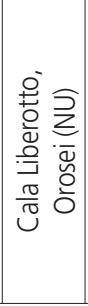 & 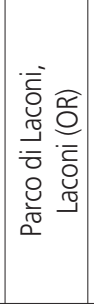 & 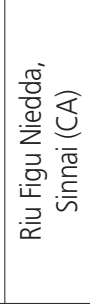 & 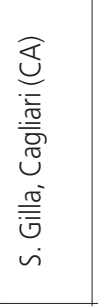 & 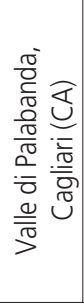 & 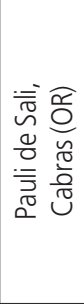 & 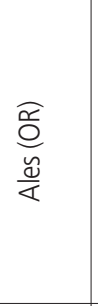 & 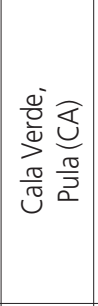 & 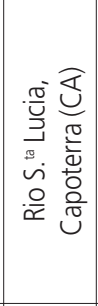 & 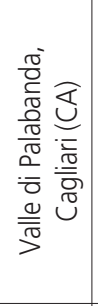 & 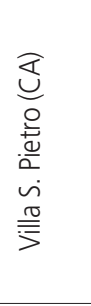 & 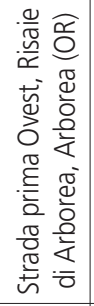 & 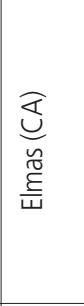 \\
\hline 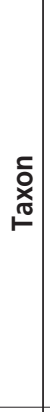 & & 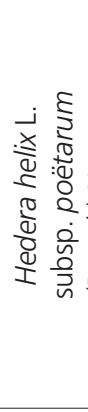 & 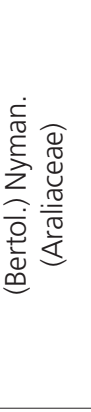 & & 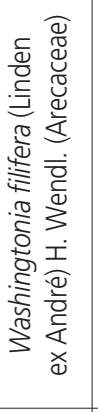 & 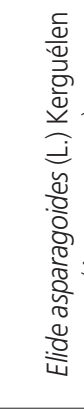 & & 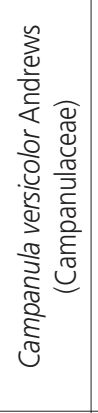 & 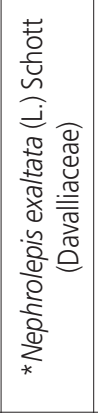 & 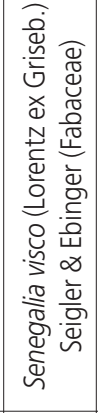 & 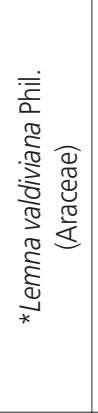 & 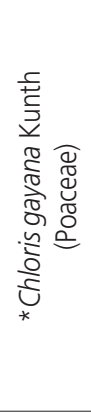 & 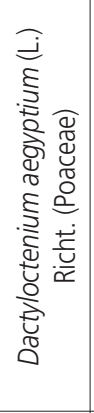 & 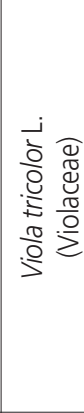 \\
\hline $\begin{array}{l}0 \\
z\end{array}$ & & $\bar{\sigma}$ & & & $\widetilde{\sigma}$ & m & & ఫ & 号 & 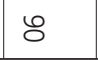 & o & $\stackrel{\infty}{\circ}$ & 8 & $\stackrel{\circ}{\circ}$ \\
\hline
\end{tabular}




\begin{tabular}{|c|c|c|c|c|c|c|c|c|c|c|c|c|c|c|c|}
\hline 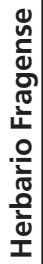 & & & & & 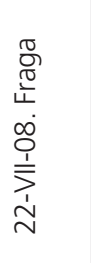 & $\begin{array}{l}\frac{\pi}{0} \\
\frac{\pi}{5} \\
0 \\
0 \\
\stackrel{1}{\overline{1}} \\
\frac{1}{0}\end{array}$ & 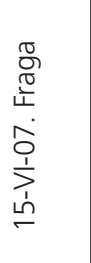 & & & & $\begin{array}{l}\frac{\pi}{0} \\
\frac{\pi}{4} \\
\dot{\omega} \\
\dot{0} \\
i \\
\dot{n} \\
\sim\end{array}$ & & & & \\
\hline 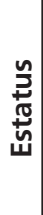 & 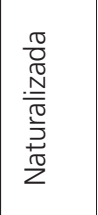 & 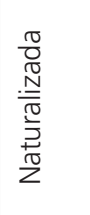 & $\begin{array}{l}\frac{0}{0} \\
\frac{\pi}{N} \\
\frac{N}{\pi} \\
\frac{2}{2} \\
\frac{\pi}{2}\end{array}$ & 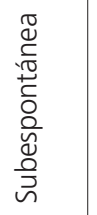 & 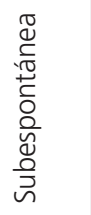 & 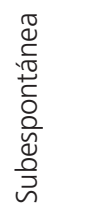 & $\begin{array}{l}\frac{\pi}{0} \\
\frac{\pi}{N} \\
\frac{N}{\pi} \\
\frac{3}{2} \\
\frac{\pi}{2} \\
\end{array}$ & $\begin{array}{l}\frac{\pi}{0} \\
\frac{N}{N} \\
\frac{N}{\pi} \\
\frac{3}{2} \\
\frac{N}{2}\end{array}$ & $\begin{array}{l}\frac{\pi}{0} \\
\frac{N}{N} \\
\frac{N}{\pi} \\
\frac{3}{2} \\
\frac{\pi}{2}\end{array}$ & 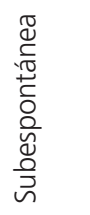 & $\begin{array}{l}\frac{\pi}{0} \\
\frac{N}{N} \\
\frac{N}{\pi} \\
\frac{N}{2} \\
\frac{\pi}{2}\end{array}$ & $\begin{array}{l}\frac{\pi}{0} \\
\frac{N}{N} \\
\frac{N}{\pi} \\
\frac{3}{2} \\
\frac{\pi}{2}\end{array}$ & $\begin{array}{l}\frac{\pi}{0} \\
\frac{N}{N} \\
\frac{N}{\sqrt[N]{0}} \\
\sum^{\frac{\pi}{2}} \\
\frac{Z}{Z}\end{array}$ & 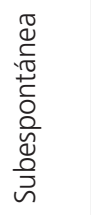 & 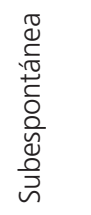 \\
\hline 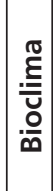 & 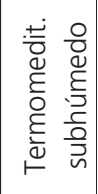 & 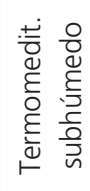 & 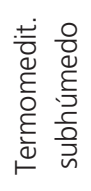 & 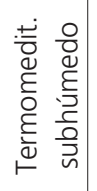 & 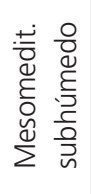 & 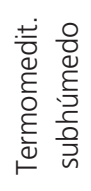 & 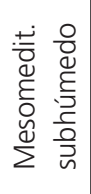 & 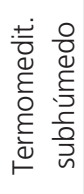 & 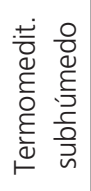 & 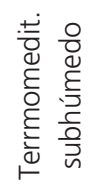 & 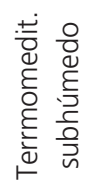 & 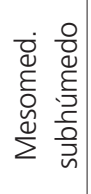 & 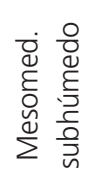 & 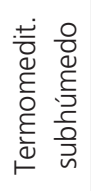 & 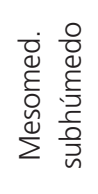 \\
\hline
\end{tabular}

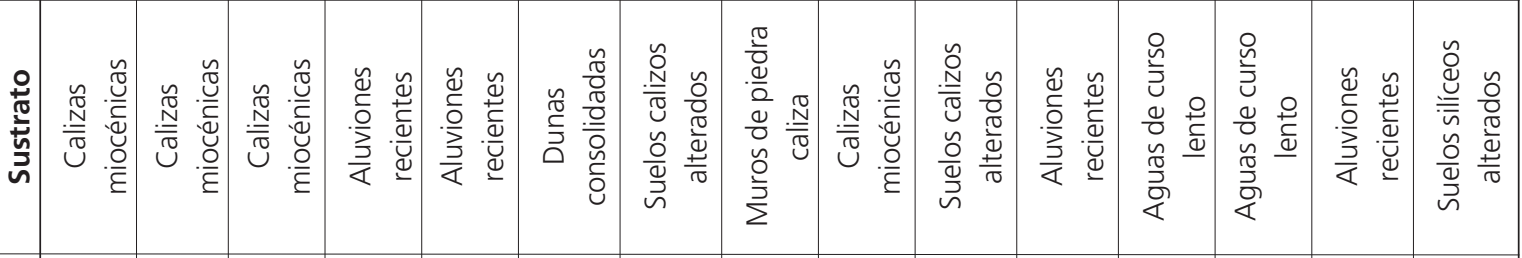

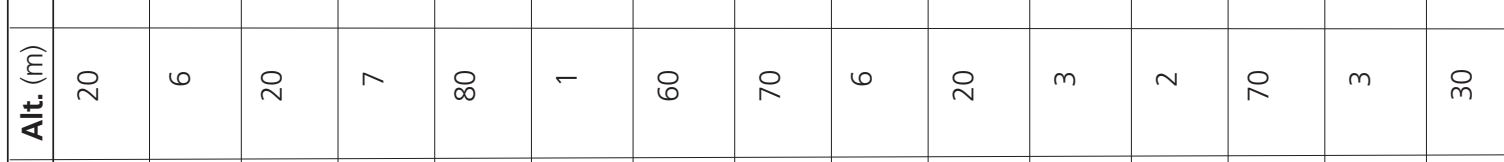

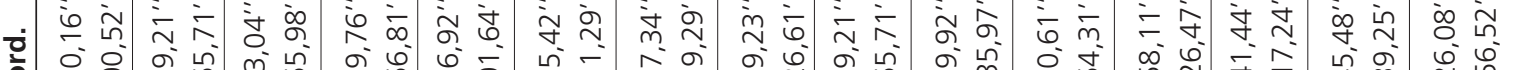

응

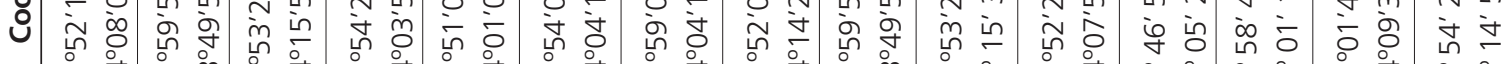

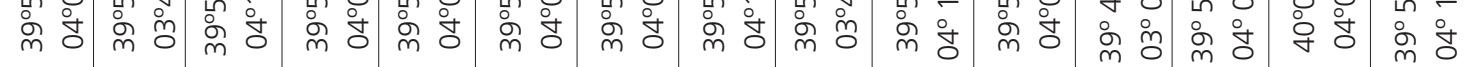

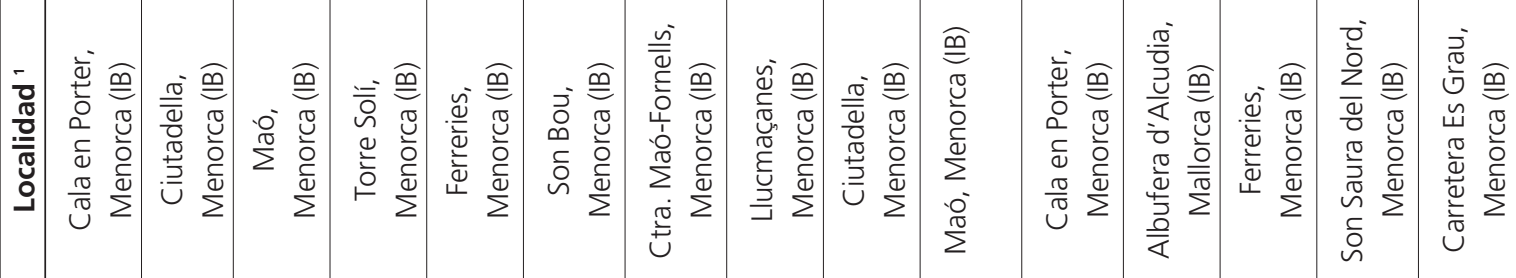

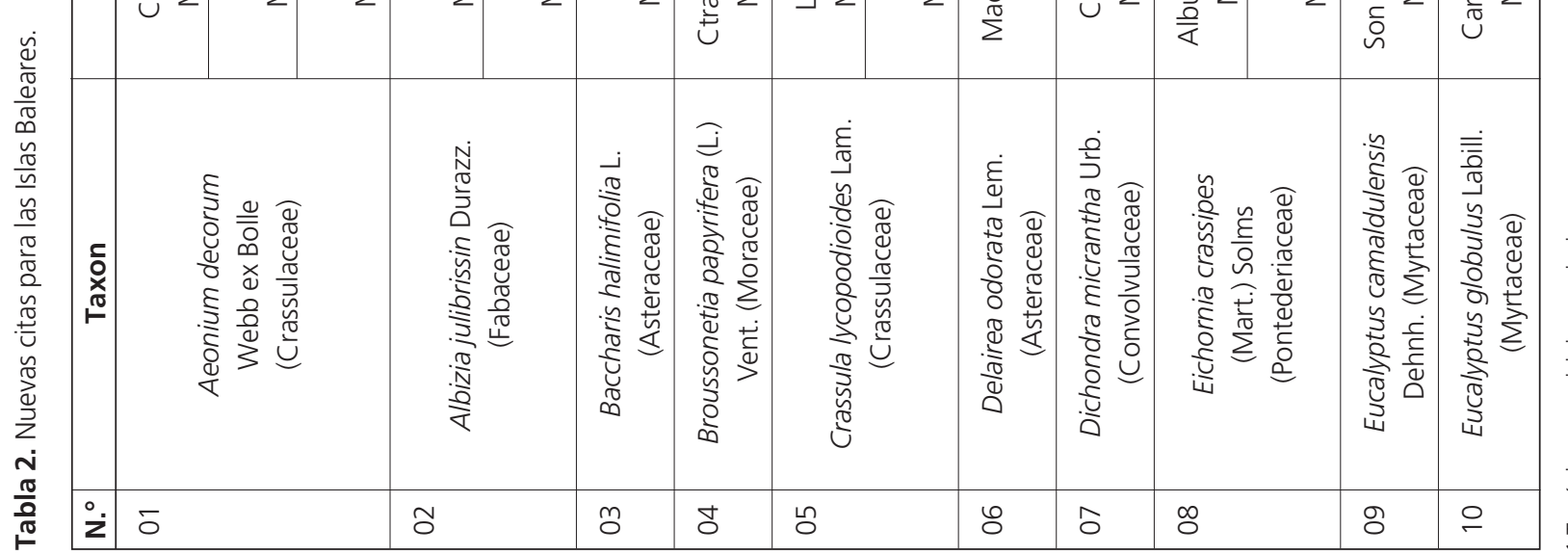




\begin{tabular}{|c|c|c|c|c|c|c|c|c|c|c|c|c|c|c|c|}
\hline$\frac{\pi}{\frac{\pi}{2}}$ & 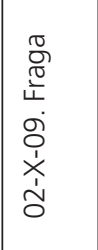 & & $\begin{array}{l}\frac{0}{0} \\
\frac{0}{4} \\
\infty \\
\infty \\
0 \\
\stackrel{1}{\overline{1}} \\
0\end{array}$ & 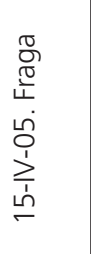 & 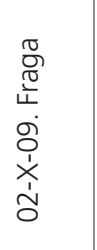 & & & & & & $\begin{array}{l}\frac{0}{0} \\
\frac{0}{0} \\
\frac{1}{4} \\
0 \\
0 \\
\frac{1}{1} \\
\frac{1}{0} \\
0\end{array}$ & & 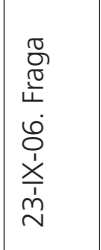 & 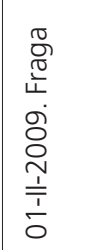 & \\
\hline & 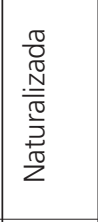 & $\begin{array}{l}\frac{\pi}{0} \\
\frac{N}{0} \\
\frac{N}{\pi} \\
\frac{2}{7} \\
\frac{\pi}{2}\end{array}$ & 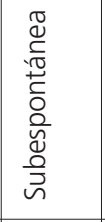 & 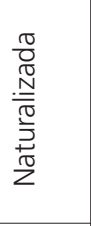 & 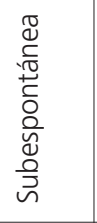 & $\begin{array}{l}\frac{\pi}{0} \\
\frac{N}{0} \\
\frac{N}{\pi} \\
\frac{0}{7} \\
\frac{\pi}{2}\end{array}$ & 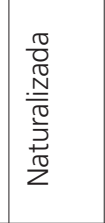 & 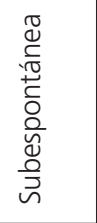 & 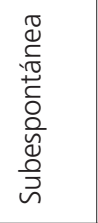 & 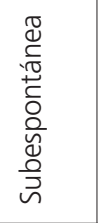 & 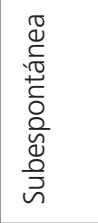 & 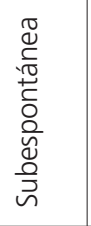 & 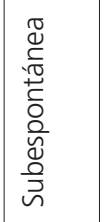 & 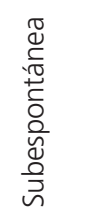 & 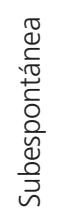 \\
\hline & 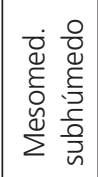 & 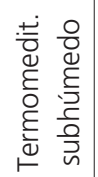 & 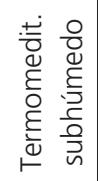 & 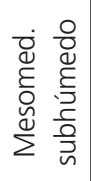 & 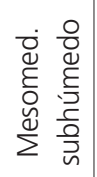 & 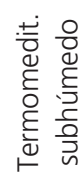 & 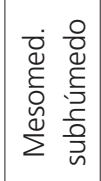 & 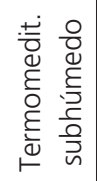 & 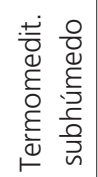 & 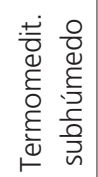 & 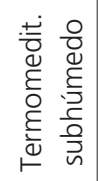 & 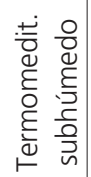 & 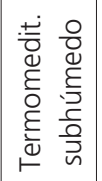 & 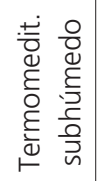 & 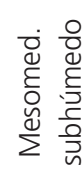 \\
\hline
\end{tabular}

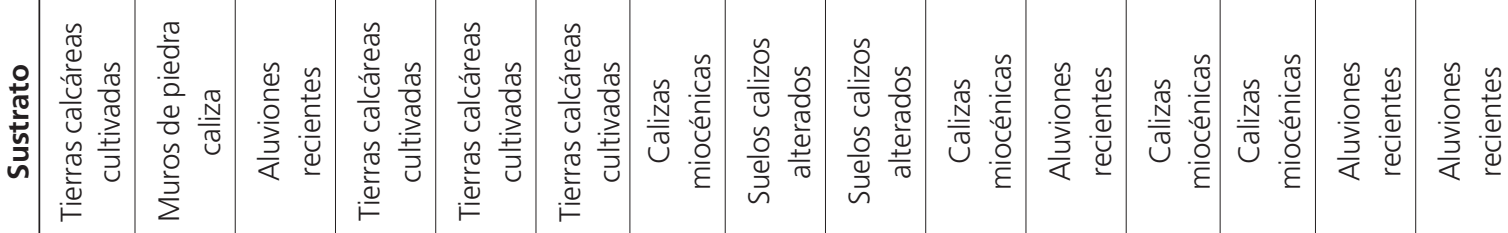

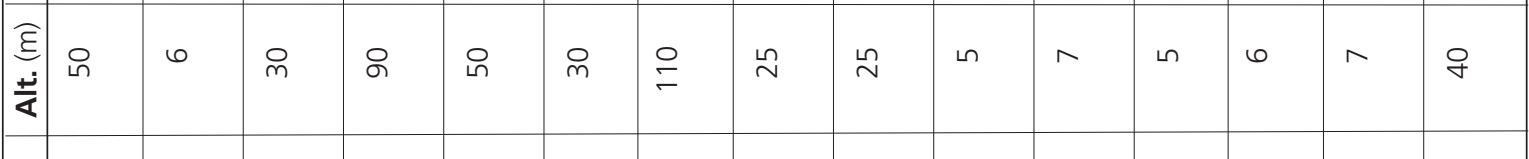

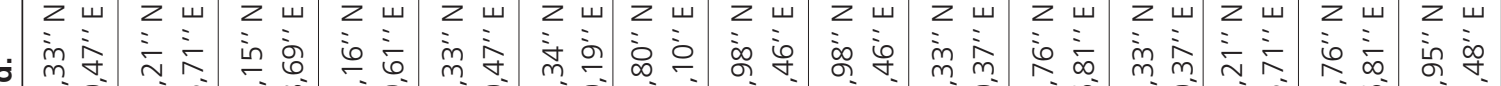

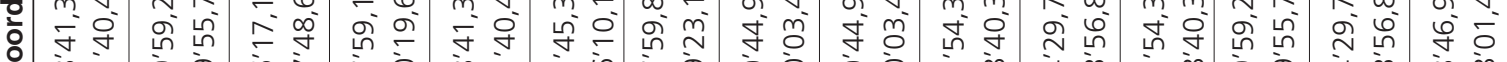

৩

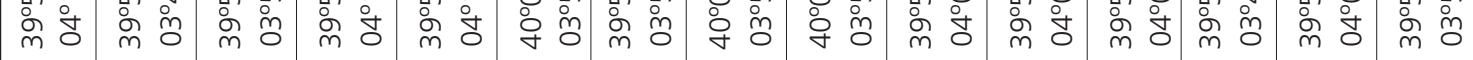

\begin{tabular}{|c|c|c|c|c|c|c|c|c|c|c|c|c|c|c|c|}
\hline 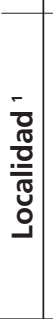 & 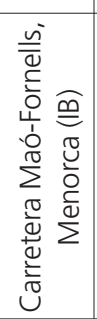 & 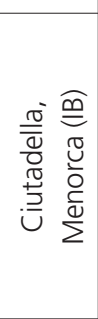 & 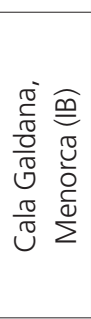 & 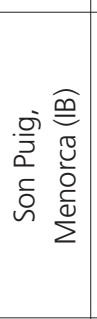 & 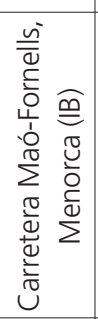 & 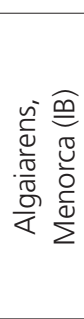 & 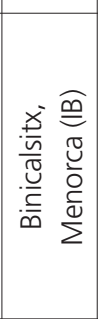 & 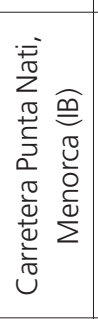 & 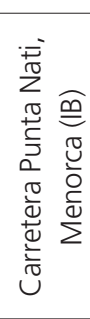 & 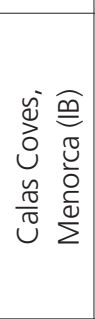 & 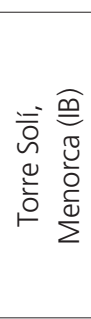 & 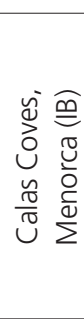 & 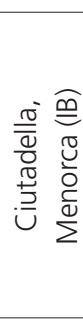 & 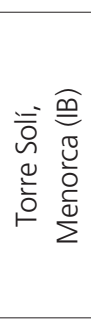 & 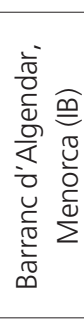 \\
\hline \begin{tabular}{|c|c} 
& \\
$x$ \\
$\sigma$ \\
$\sigma$
\end{tabular} & 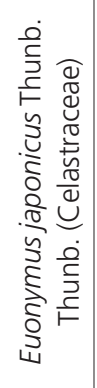 & 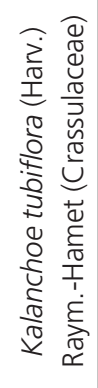 & 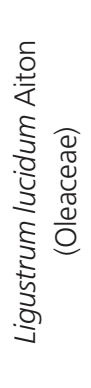 & 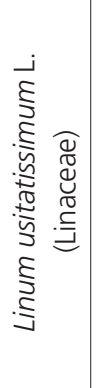 & 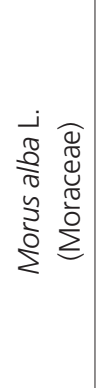 & 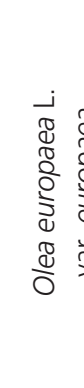 & 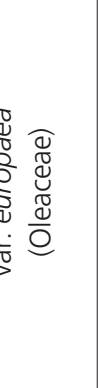 & 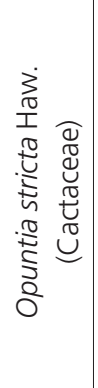 & 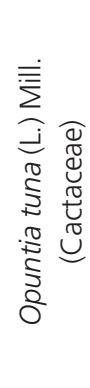 & 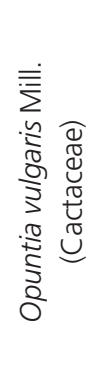 & 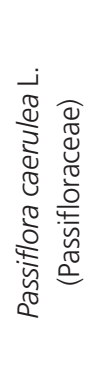 & $\begin{array}{l}\frac{5}{3} \\
\overline{0} \\
\frac{0}{0} \\
\frac{0}{2} \\
0\end{array}$ & & 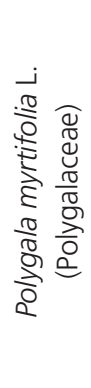 & 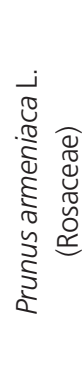 \\
\hline$\dot{z}$ & $\mp$ & $\simeq$ & $\underline{m}$ & $\underset{+}{ \pm}$ & $\stackrel{\Perp}{\sim}$ & $\stackrel{\circ}{\circ}$ & & 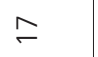 & $\stackrel{\infty}{-}$ & $\stackrel{g}{-}$ & ㅇ & $\bar{\sim}$ & & $\approx$ & $\stackrel{m}{\sim}$ \\
\hline
\end{tabular}




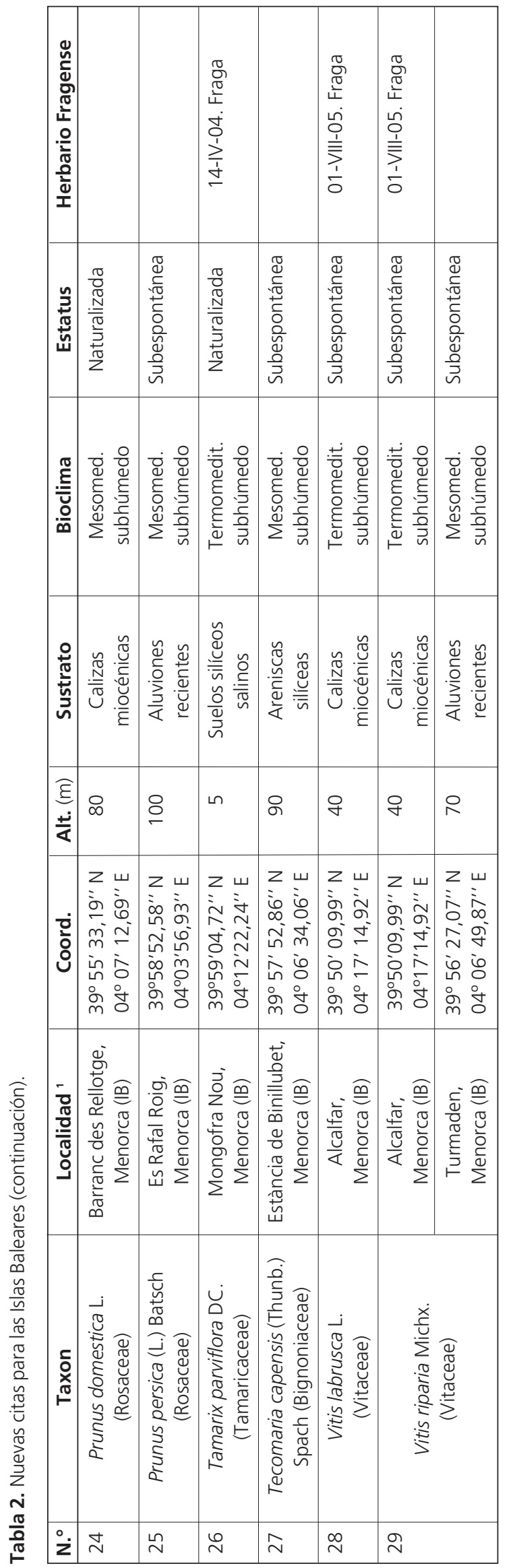

Los neófitos y arqueófitos suponen el 61\% y 39\% de la flora exótica, tanto de Cerdeña como de las Baleares. La proporción de neófitos naturalizados es del $26 \%$ (SA) y 28\% (BL), de neófitos subespontáneos del $24 \%$ (SA) y $20 \%$ (BL), y de neófitos invasores del $11 \%$ (SA) y $13 \%$ (BL). Atendiendo a los arqueófitos, el porcentaje de naturalizados es del $20 \%$ (SA) y $24 \%$ (BL), de subespontáneos del 18\% (SA) y 14\% (BL) y de invasores del 1\% para ambos territorios (Fig. 3). El test de Mann-Whitney ha confirmado que no hay diferencias significativas entre las medianas de las mues$\operatorname{tras}(\mathrm{U}=2, p=1,000)$.

La comparación entre el espectro biológico de los dos territorios (Fig. 4) revela que en Cerdeña los fanerófitos son el tipo más representado (40\% SA, 32\% $\mathrm{BL})$, mientras los terófitos prevalecen en las Baleares (30\% SA, 34\% BL), seguidos por los hemicriptófitos (13\% SA, 16\% BL), geófitos (9\% SA, 10\% BL), caméfitos ( $6 \%$ SA, $7 \%$ BL) e hidrófitos ( $2 \%$ SA, $1 \%$ BL). El test de Mann-Whitney ha evidenciado que no existen diferencias significativas entre las medianas de las muestras ( $\mathrm{U}=6, p=0,9362)$.

Un análisis de los datos atendiendo al origen geográfico (Fig. 5) muestra una dominancia del elemento americano (32\% SA, 32\% BL) sobre el mediterráneo (17\% SA, 20\% BL), seguidos del asiático (11\% SA, $11 \%$ BL), sáharo-arábigo (10\% SA, 7\% BL), capense ( $8 \%$ SA, $10 \%$ BL) y euroasiático (4\% SA, 7\% BL); menor relevancia tienen los elementos europeo, australiano y africano. El test de Mann-Whitney ha revelado que no hay diferencias significativas entre las medianas de las muestras $(U=10, p=0,737)$. El análisis del origen de las especies invasoras (Fig. 6) confirma asimismo el dominio del elemento americano (61\% SA, 57\% BL) sobre el resto (39\% SA, 43\% BL). Le sigue el elemento capense (19\% SA, 23\% BL) y asiático $(6 \% \mathrm{SA}, 8 \% \mathrm{BL})$, mientras que el mediterráneo posee un bajo porcentaje ( $4 \% \mathrm{SA}, 4 \% \mathrm{BL}$ ). El test de Mann-Whitney ha mostrado que no hay diferencias significativas entre las medianas de las muestras $(\mathrm{U}=$ $10, p=0,8102$ ).

El análisis de las vías de introducción (Fig. 7) muestra que la mayoría de las especies han sido introducidas por el ser humano de forma intencionada en ambos territorios (76\% SA, 77\% BL). El uso ornamental explica la mayor parte de estas introducciones $(43 \%$ $\mathrm{SA}, 45 \% \mathrm{BL}$ ), seguido de las especies introducidas para usos agrícolas $(25 \% \mathrm{SA}, 32 \% \mathrm{BL})$, de las infestantes (16\% SA, 16\% BL), accidentales ( $8 \%$ SA, $7 \% \mathrm{BL})$ y de las introducidas para selvicultura $(8 \%$ SA, $0 \%$ BL). El test de Mann-Whitney ha confirmado que no hay diferencias significativas entre las medianas de las muestras ( $U=5, p=0,8345)$. 
Con el fin de verificar el impacto de las especies exóticas sobre los ecosistemas, se ha realizado un análisis comparativo en relación con los hábitats más sensibles. Para Cerdeña resultan mayoritariamente sujetos a fenómenos de colonización las zonas agrícolas $(30 \%)$ y sinantrópicas $(30 \%)$, seguidas por los ecosistemas húmedos $(11 \%)$, litorales $(10 \%)$ y riparios $(8 \%)$. Por su parte, los ecosistemas más afectados en las Baleares son los sinantrópicos (45\%) y secundariamente los agrícolas (28\%), seguidos por los riparios $(8 \%)$, litorales $(8 \%)$ y los de zonas húmedas $(7 \%)$ (Fig. 8). El test de Mann-Whitney ha confirmado que no hay diferencias significativas entre las medianas de las muestras $(\mathrm{U}=7, p=0,4433)$.

Para Cerdeña, el porcentaje mayor de especies invasoras en los hábitats más sensibles se da en los ecosistemas litorales (24\%; 11 táxones), seguido de las zonas húmedas $(15 \% ; 8)$, hábitats arbustivos $(11 \%$; $2)$, riparios $(10 \% ; 4)$, agrícolas-rurales $(10 \% ; 14)$ y sinantrópicos $(10 \% ; 14)$. No se han encontrado especies invasoras en los hábitats forestales (Fig. 9). El test de Mann-Whitney ha revelado que existen diferencias altamente significativas entre las medianas de las muestras ( $U=7, p<0,01)$. Por su parte, en las Baleares los hábitats con una mayor presencia de invasoras son las zonas húmedas (36\%; 9 táxones), seguidos por los litorales $(28 \% ; 8)$, agrícolas-rurales $(16 \% ; 16)$, riparios $(14 \% ; 4)$ y sensiblemente menor los sinantrópi$\cos (7 \% ; 10)$. En los ambientes forestales sólo se ha identificado un taxon invasor, mientras que no aparece ninguno en los de bosque bajo (hábitats arbustivos). El test de Mann-Whitney ha confirmado que existen diferencias significativas entre las medianas de las muestras $(\mathrm{U}=7, p<0,05)$.

Tabla 3. Comparación numérica de cada tipo de exótica respecto al total de especies comunes entre Cerdeña y Baleares.

\begin{tabular}{|l|c|c|c|r|}
\hline \multirow{2}{*}{} & \multicolumn{2}{|c|}{ Total (número) } & \multicolumn{2}{c|}{ Comunes total (número) } \\
\cline { 2 - 5 } & Cerdeña & Baleares & Cerdeña & Baleares \\
\hline Naturalizadas & 217 & 180 & 128 & 114 \\
Subespontáneas & 202 & 119 & 78 & 92 \\
Invasoras & 53 & 48 & 40 & 40 \\
\hline
\end{tabular}

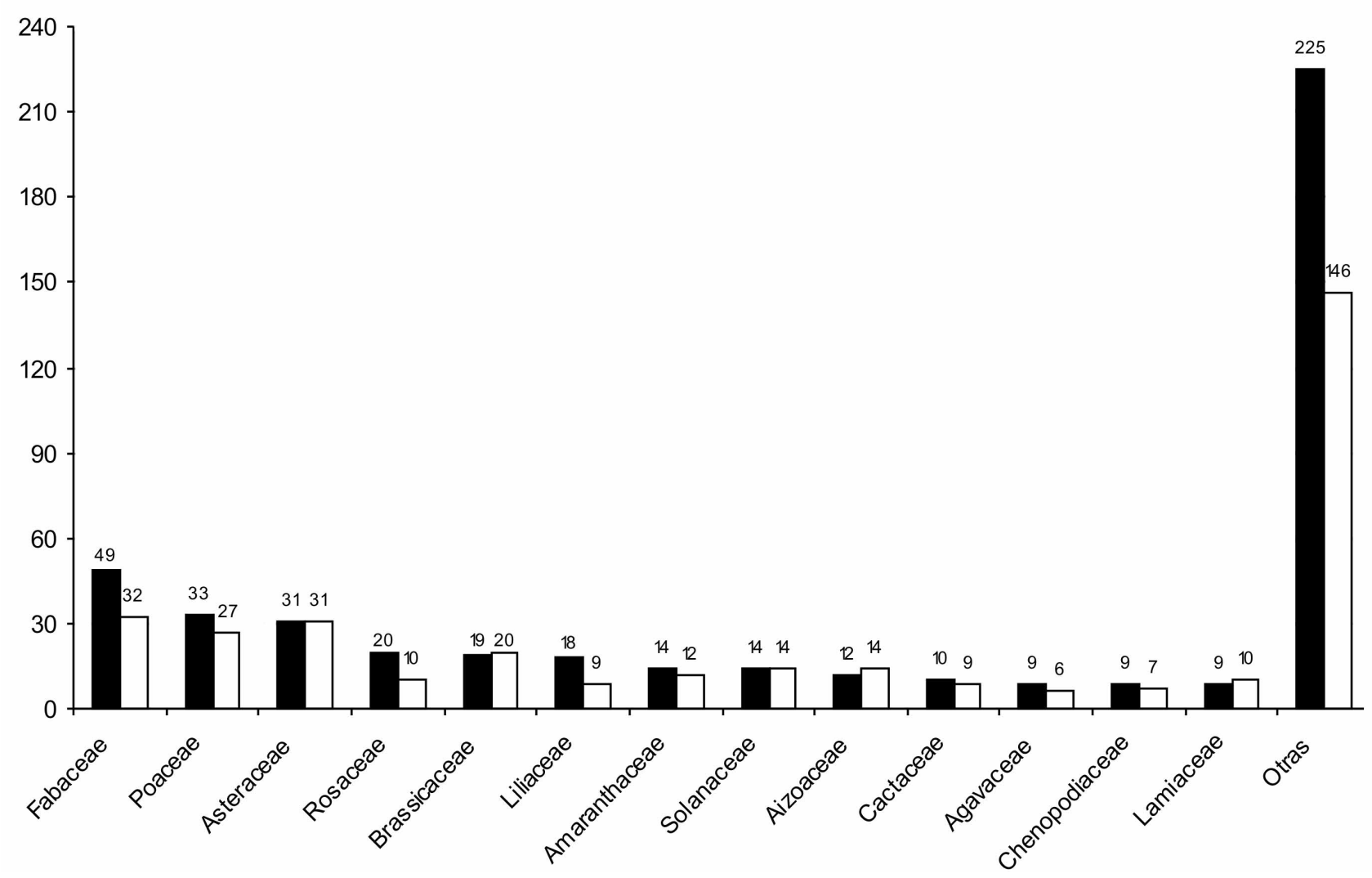

Fig. 2. Comparación del número de especies por familias en Cerdeña (negro) y las Baleares (blanco). 


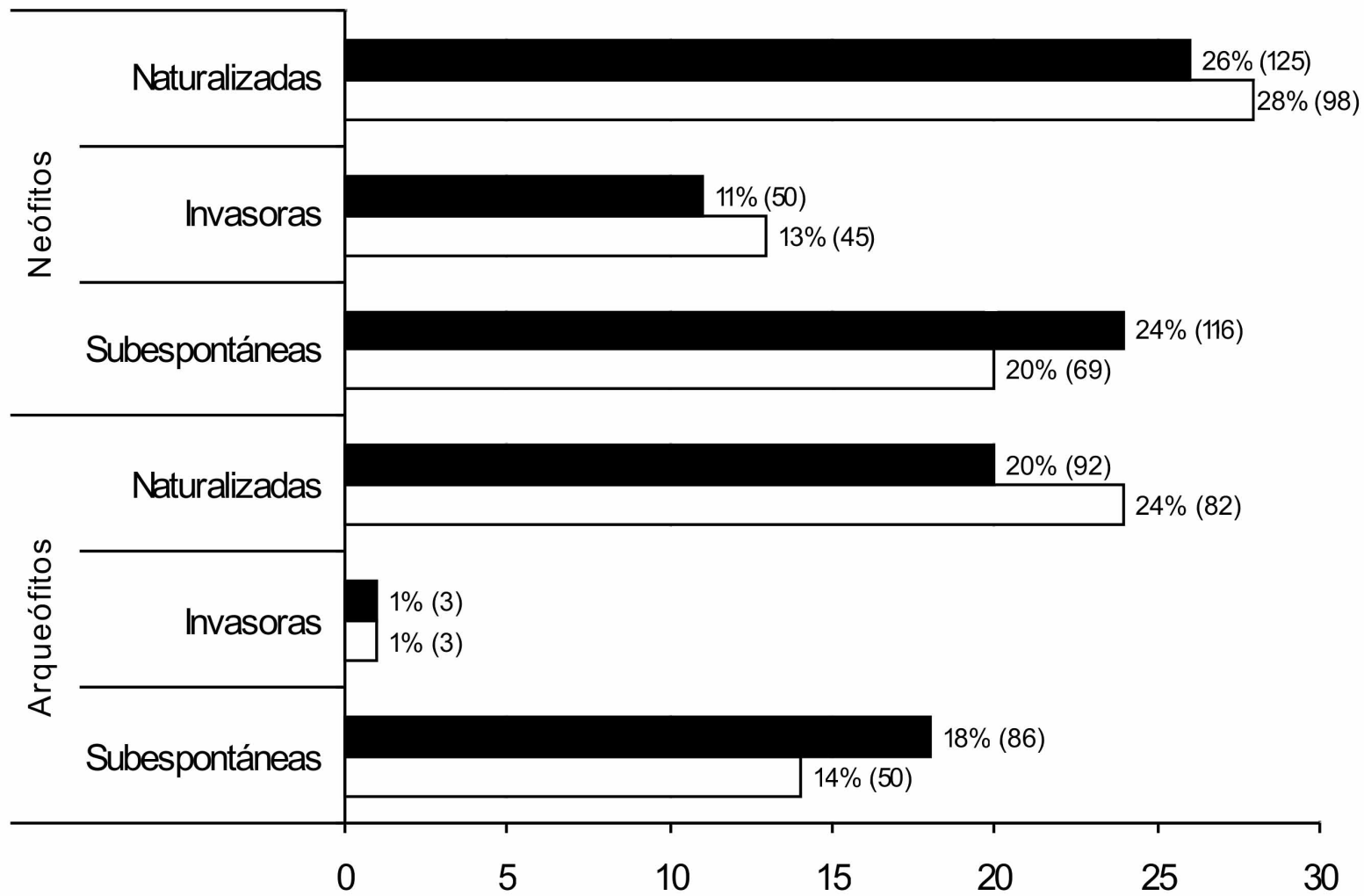

Fig. 3. Comparación porcentual y numérica de cada tipo de alóctona, diferenciando entre arqueófitos y neófitos, respecto al total de flora exótica de Cerdeña (negro) y las Baleares (blanco).

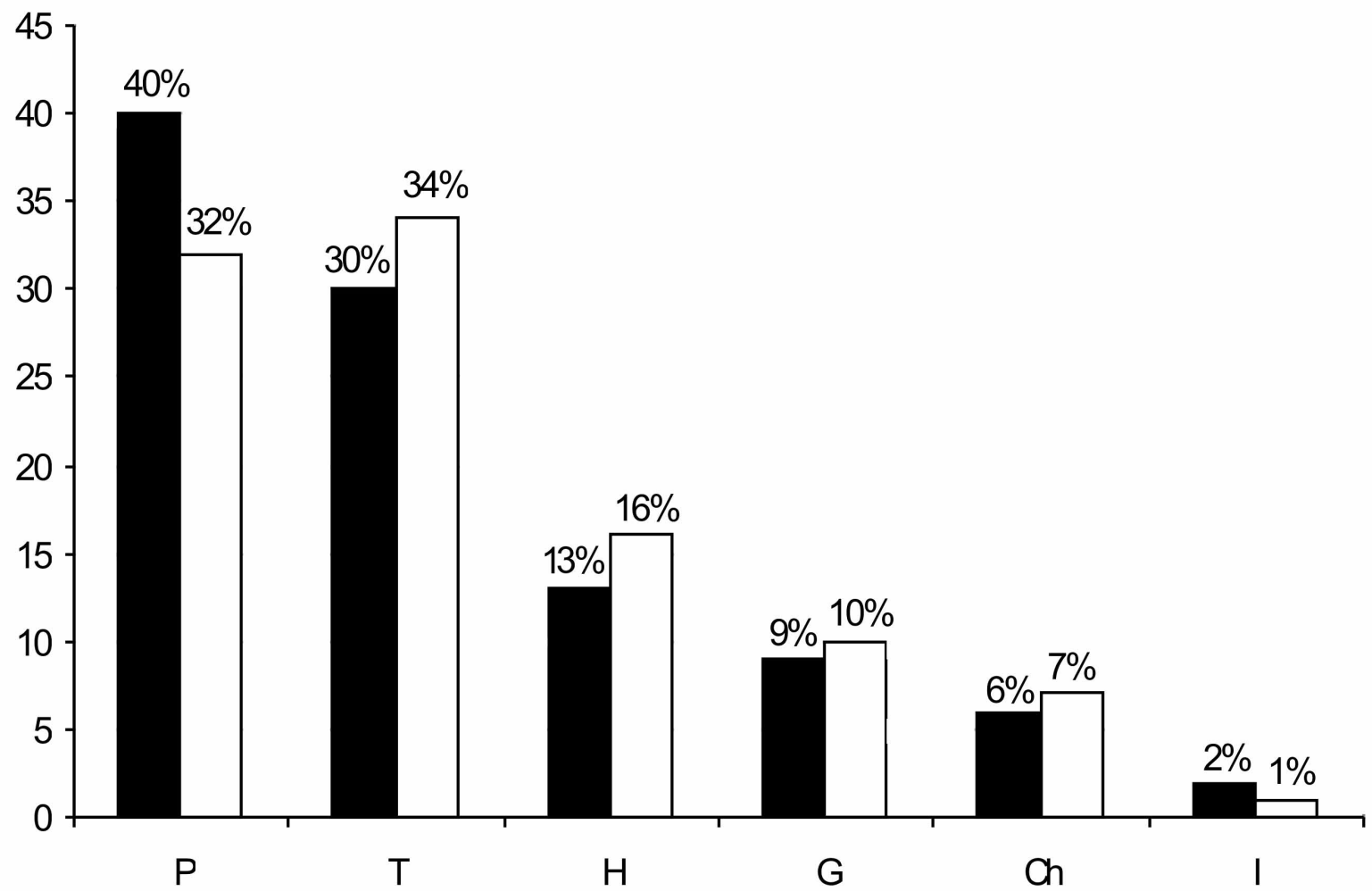

Fig. 4. Comparación porcentual entre las formas de vida de la flora exótica de Cerdeña (negro) y las Baleares (blanco): P, fanerófitos; T, terófitos; $\mathbf{H}$, hemicriptófitos; G, geófitos; $\mathbf{C h}$, caméfitos; I, hidrófitos. 


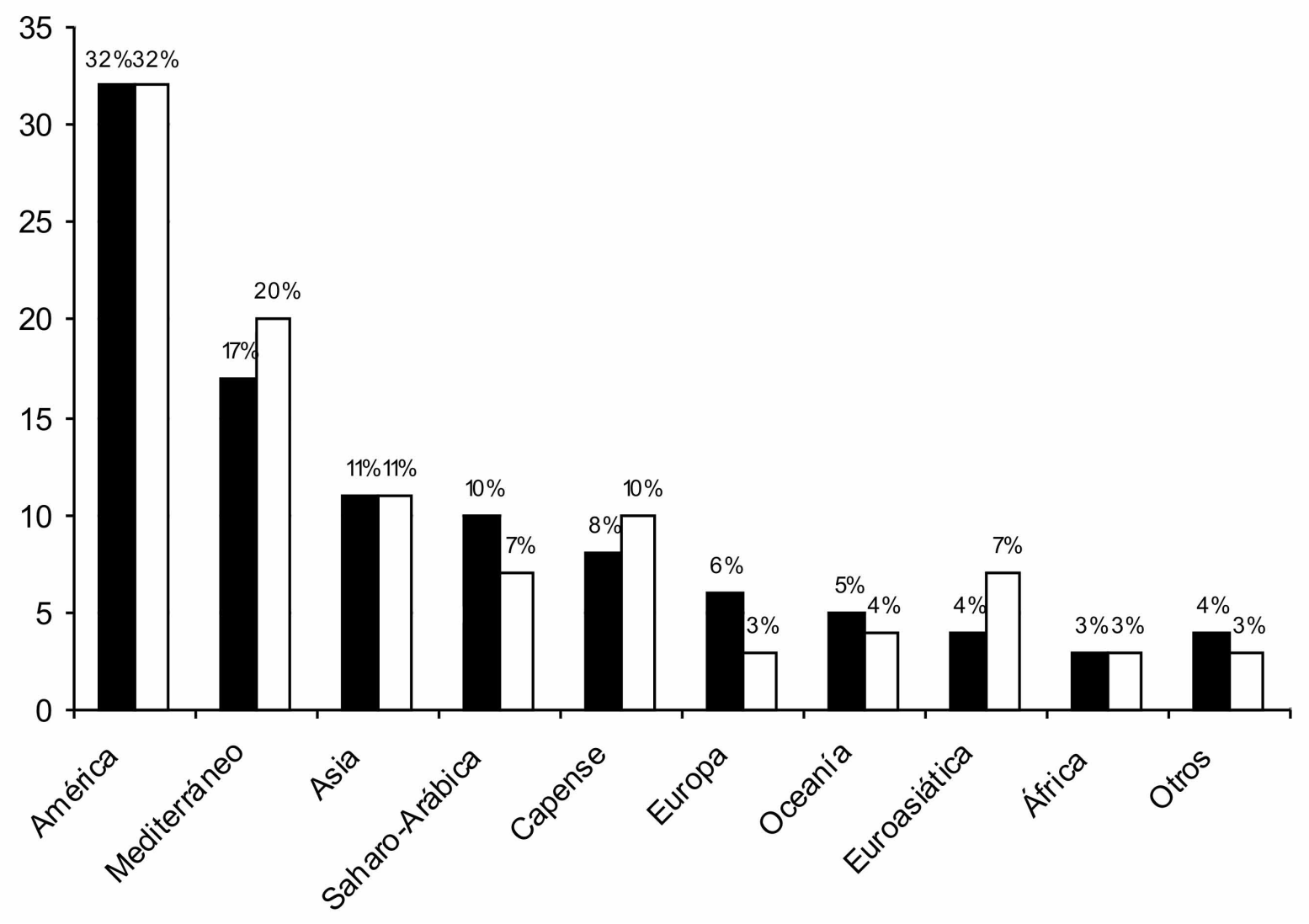

Fig. 5. Comparación porcentual entre el origen geográfico de la flora exótica de Cerdeña (negro) y las Baleares (blanco).

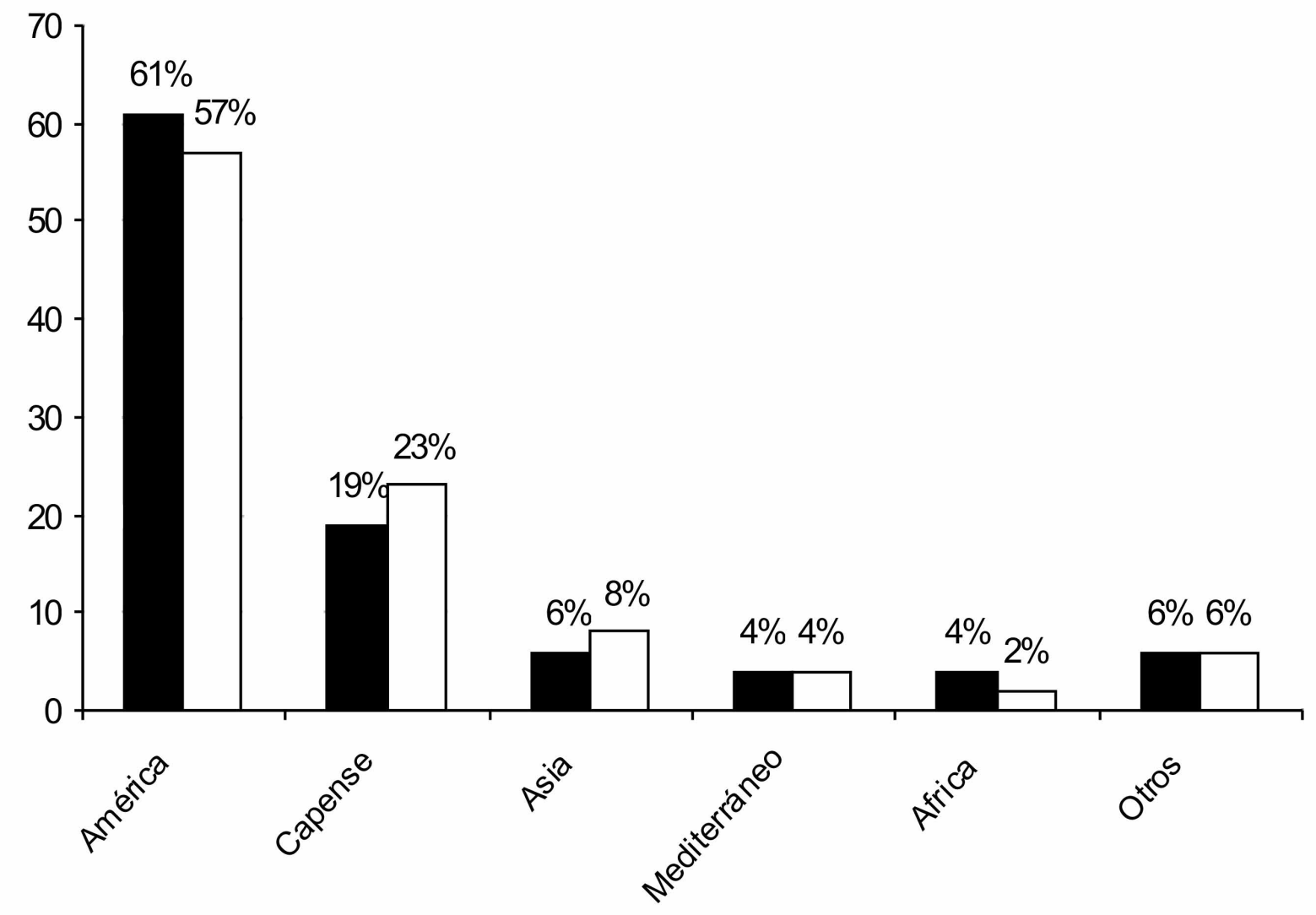

Fig. 6. Comparación porcentual de las invasoras según el origen geográfico de la flora exótica de Cerdeña (negro) y las Baleares (blanco). 


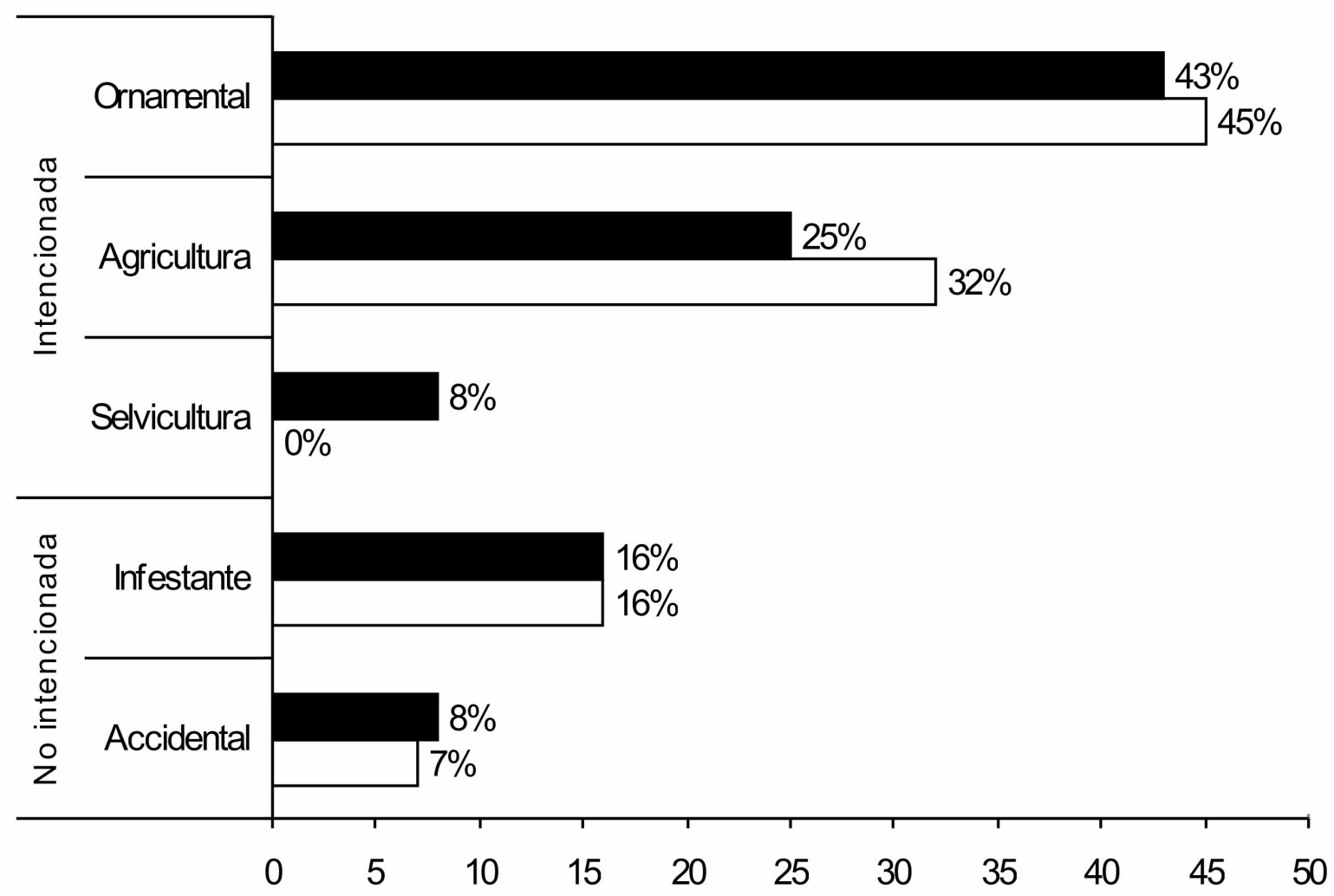

Fig. 7. Comparación porcentual de las vías de introducción de la flora exótica de Cerdeña (negro) y las Baleares (blanco).

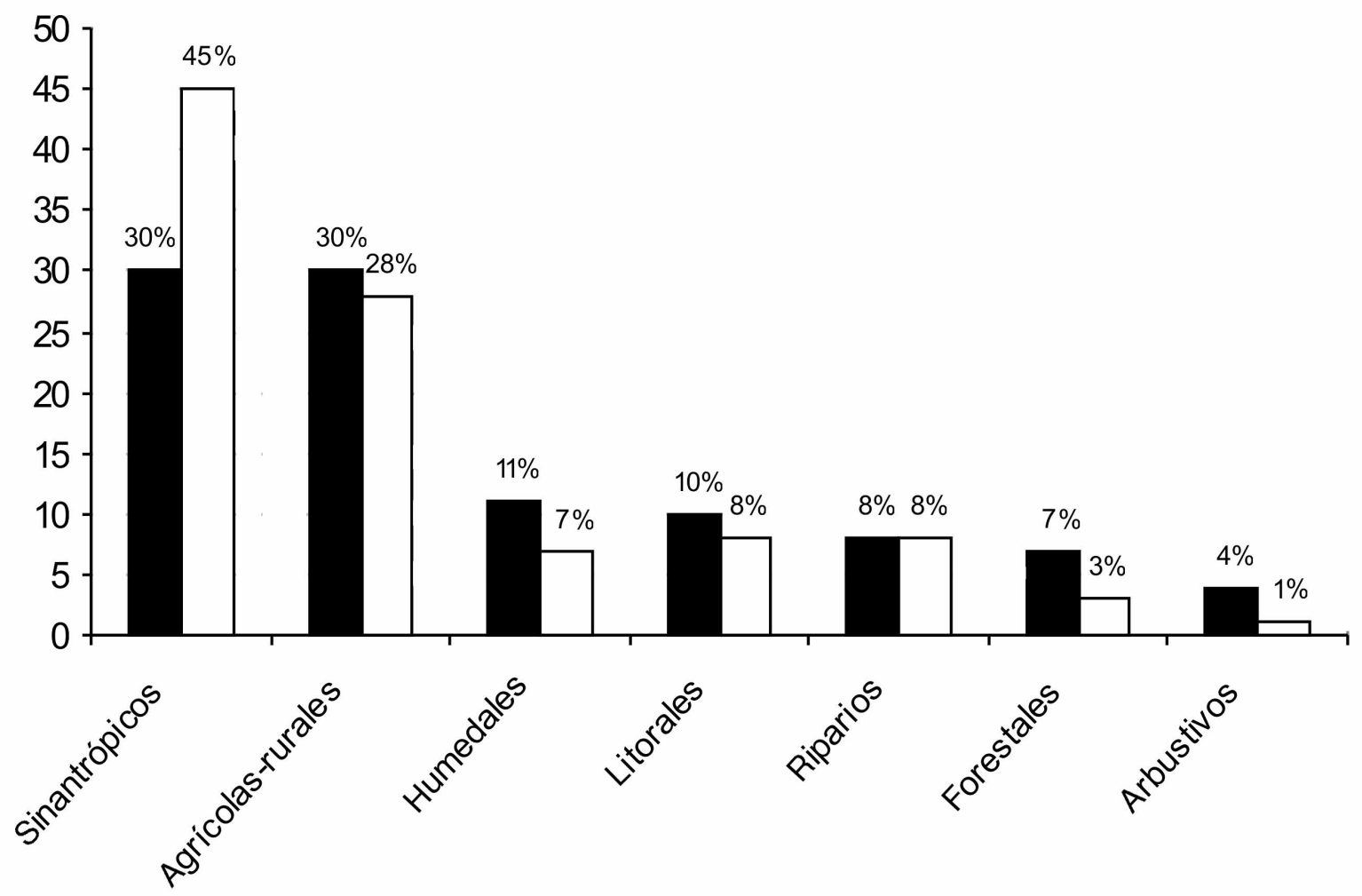

Fig. 8. Comparación porcentual de los hábitats afectados por la flora exótica de Cerdeña (negro) y las Baleares (blanco). 


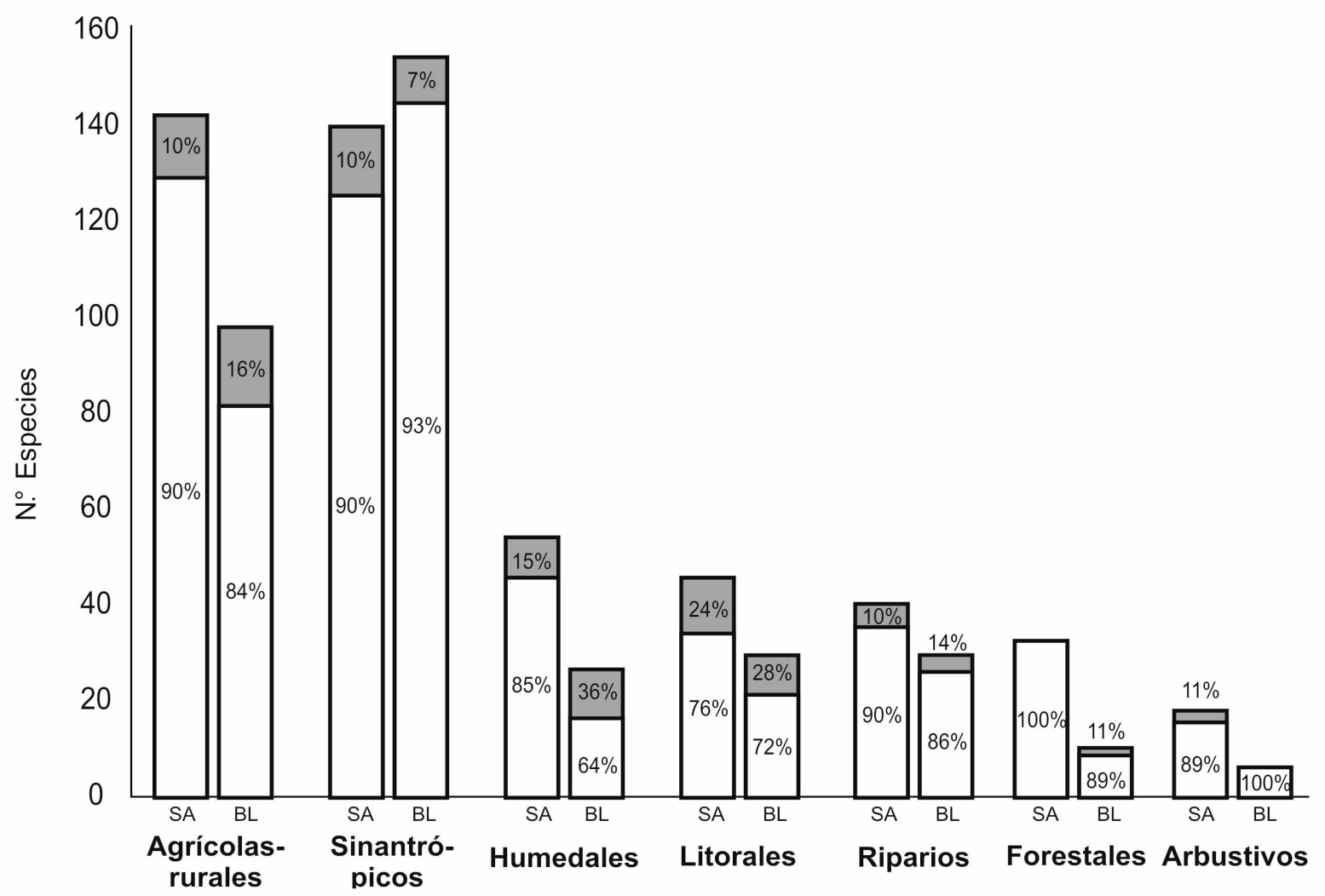

Fig. 9. Comparación numérica y porcentual de especies invasoras (gris) respecto a la suma de las especies naturalizadas y subespontáneas (blanco) de la flora exótica en función de los hábitats que ocupan en Cerdeña y en las Baleares.

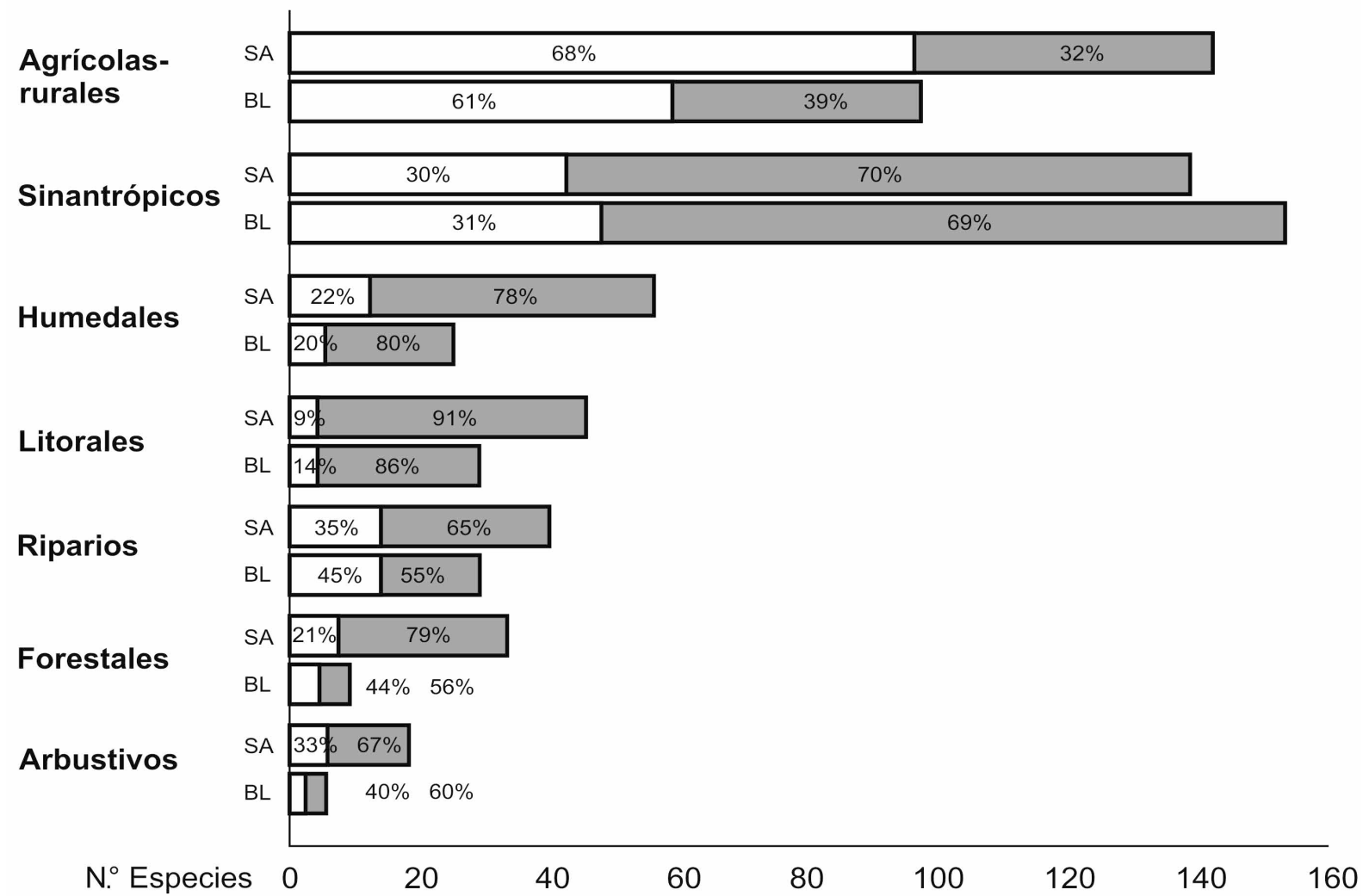

Fig. 10. Comparación porcentual de especies de arqueófitos (blanco) y neófitos (gris) en función de los hábitats que ocupan en Cerdeña y en las Baleares. 
También se han efectuado comparaciones entre los porcentajes de arqueófitos y neófitos en estos hábitats para Cerdeña y para las Islas Baleares (Fig. 10). Los hábitats más ricos en arqueófitos son los agrícolas-rurales $(68 \% \mathrm{SA}, 61 \% \mathrm{BL})$, mientras que los más ricos en neófitos son los litorales (91\% SA, 86\% BL). Todos los otros hábitats poseen porcentajes considerablemente menores de arqueófitos respecto a neófitos. Para los hábitats riparios los porcentajes de arqueófitos son del $35 \%$ (SA) y $45 \%$ (BL), para los arbustivos del $33 \%$ (SA) y $40 \%$ (BL), para los sinantrópicos del $30 \%(\mathrm{SA})$ y $31 \%$ (BL), para las zonas húmedas del $22 \%$ (SA) y $20 \%$ (BL), para los forestales del $21 \%$ (SA) y $44 \%(\mathrm{BL})$, y para los litorales tienen valores más bajos ( $9 \%$ SA, 14\% BL). El test de Mann-Whitney ha confirmado que no existen diferencias significativas entre las medianas de las muestras (SA: $\mathrm{U}=7$, $p=0,1094$; BL: $\mathrm{U}=7, p=0,4052$ ).

\section{Discusión}

Las similitudes de la flora exótica de los dos ámbitos de estudio resultan estadísticamente significativas, lo que confirma que territorios insulares continentales que se encuentran en la misma región biogeográfica, con unas características ambientales (paleogeográficas, geográficas, climáticas, bioclimáticas y biogeográficas) similares, se pueden comparar y muestran una respuesta similar ante la invasión por parte de especies de flora exótica.

De hecho, ambos territorios tienen en común un número importante de táxones exóticos (246) y poseen el mismo porcentaje de invasoras en común $(16 \%)$. Entre ellos, algunos son considerados invasores en todo el mundo [p. ej., Ailantbus altissima (Mill.) Swingle, Arundo donax L., Carpobrotus edulis (L.) N.E. Br., Cortaderia selloana (Schult. \& Schult. f.) Asch. \& Graebn., Nicotiana glauca R.C. Graham, Oxalis pes-caprae L., Paspalum distichum (Michx.) Scrib., Pennisetum setaceum (Forssk.) Chivo., Xanthium spinosum L.] (Weber, 2003) y en Europa [A. altissima, C. edulis, C. selloana, O. pes-caprae, P. distichum] (DAISIE, 2009). A excepción de C. selloana y $P$. setaceum, todos los demás táxones han sido reconocidos como los más invasores en las islas del Mediterráneo (Lloret \& al., 2004; Hulme \& al., 2008b). Además, algunos invasores exclusivos de las Baleares, como Canna indica L., Echinochloa sp. pl. o Ipomoea indica (Burm.) Merr., en Cerdeña están todavía en el estatus de naturalizados, o incluso subespontáneos, como es el caso de Lonicera japonica Thunb. En cambio, especies invasoras en Cerdeña como Agave americana L., Opuntia sp. pl. o Acacia saligna (Labill.) H. Wendl., en las Baleares presentan un estatus de natu- ralizada, o subespontánea (p. ej., Solanum elaeagnifolium Cav.).

La flora naturalizada muestra porcentajes levemente diferentes en ambos territorios en cuanto al proceso de naturalización. Considerando los táxones exóticos comunes a ambos territorios, se observa que las especies naturalizadas tienen más importancia en Cerdeña que en Baleares. En cambio, la tendencia se invierte en las subespontáneas. Estos resultados pueden indicar que por un lado la mayor superficie de Cerdeña y por otro una mayor diversidad en factores como la orografía o la geología generan más hábitats favorables a la naturalización. Sin embargo, en las Baleares, debido a la mayor presión antrópica (152 hab $/ \mathrm{km}^{2}$ vs. $69 \mathrm{hab} / \mathrm{km}^{2}$ de Cerdeña), el proceso de introducción de especies sería más activo y dinámico, una situación que favorece el mayor porcentaje de subespontáneas, probablemente resultado de introducciones recientes. El porcentaje de invasoras, similar en ambos territorios, muestra la misma tendencia. De hecho, las Islas Baleares, al sufrir una mayor presión antrópica, tienen proporcionalmente una mayor presencia de invasoras que Cerdeña a pesar de que este último territorio presentaría más situaciones favorables para la expansión de éstas. Estos datos pueden resultar de gran utilidad desde un punto de vista práctico con vistas a la gestión de esta problemática. A causa de la similitud entre ambos territorios, las plantas que en Baleares están en proceso de naturalización o se comportan ya como invasoras, tienen muchas probabilidades de desarrollar una tendencia similar en Cerdeña.

Un examen de la situación de los arqueófitos de las dos floras exóticas muestra que aquellas especies introducidas antes de 1500 son bastante similares en los dos territorios, mientras que se encuentran diferencias en los neófitos. En este sentido, es importante destacar que los arqueófitos invasores tienen poca influencia, suponiendo un porcentaje mínimo respecto al total, tanto para Cerdeña como para las Baleares. De hecho, Ricinus communis L., A. donax y Acanthus mollis L. para Cerdeña y A. donax, Brassica rapa L., Spartium junceum L. para Baleares son los únicos arqueófitos invasores. En contraste, los neófitos invasores suponen en ambos territorios un alto porcentaje.

La comparación del espectro biológico de ambas áreas muestra diferencias en el porcentaje de fanerófitos, que es mayor en Cerdeña, mientras que para los terófitos es mayor en las Baleares. La diferencia de terófitos puede explicarse por la ya citada menor naturalidad debida a la mayor actividad humana de las Baleares y a la elevada densidad poblacional, que fa- 
vorecería la presencia de esta forma biológica por su mejor adaptación a los ambientes alterados (Madon \& Médail, 1997; Schippers \& al., 2001; Hill \& al., 2002; Kotanen, 2004), mientras que el predominio de los fanerófitos en Cerdeña respondería a una mayor utilización de especies exóticas leñosas en las plantaciones y reforestaciones.

El análisis de los datos atendiendo al origen geográfico muestra una dominancia del elemento americano como ocurre en muchas floras exóticas del sur de Europa y norte de África, especialmente en los países del Mediterráneo (Vilà \& al., 1999; Sanz Elorza \& al., 2004; Bazos \& al. 2009; Celesti-Grapow \& al., 2009b; Dal Cin D'Agata \& al., 2009). Por otra parte, el mayor porcentaje de especies invasoras es de origen americano, seguido del capense. Estos datos confirman la mayor capacidad de adaptación de estos táxones al clima mediterráneo, que se ve facilitado por la dispersión de propágulos en el medio ambiente como consecuencia del frecuente uso de la propagación vegetativa con fines ornamentales (Lockwood \& al., 2005). Por otra parte, también está asociada con el abandono no autorizado de restos de plantas con propágulos en zonas desde donde se naturalizan con facilidad. Este fenómeno está subestimado en la actualidad en ambos territorios insulares y realmente sólo se ha tenido en cuenta en la Comunidad Valenciana, donde está sancionado por el Decreto 213/2009 de 20 de noviembre de la Conselleria de Medio Ambiente, Agua, Urbanismo y Vivienda de la Generalitat Valenciana, por el que se aprueban medidas para el control de las especies vegetales exóticas invasoras (Comunitat Valenciana, 2009).

Los resultados del análisis de las vías de introducción resultan especialmente útiles para la prevención y el establecimiento de estrategias de control (Wittenberg \& Cock, 2001; Hulme, 2006, 2007; García-Berthou \& al., 2008), evidenciando el importante papel que ha desempeñado la actividad humana a lo largo del tiempo en el establecimiento de la flora exótica en ambos territorios. Actualmente las especies introducidas con fines ornamentales son las más abundantes como consecuencia de la proliferación en zonas costeras de jardinería mediterránea basada en el uso de especies provenientes de áreas de climas análogos. (Hulme \& al., 2008b). El porcentaje de especies introducidas para uso silvícola es nulo en las Baleares debido al escaso empleo de esta práctica en ese archipiélago. Aunque, como han señalado otros autores (Hulme \& al., 2008a; Lambdon \& al., 2008; Pyšek \& al., 2009), las introducciones son principalmente intencionadas, no se han de minusvalorar las no intencionadas, como la introducción accidental de semillas. En este sentido, el número de especies accidentales, que resulta bajo, podría estar subestimado debido a la dificultad técnica de encontrar los datos de este tipo de introducción (Mack, 2000).

El análisis de los hábitats más sensibles muestra muchas similitudes entre ambas zonas, con una tendencia general a que los hábitats más colonizados por especies exóticas sean los seminaturales, agrícolas y sinantrópicos, resultado previsible, dada la fuerte presión y las continuas perturbaciones causadas por el hombre. Sin embargo, el porcentaje de especies invasoras no es elevado y no es comparable al grado de invasión que sufren los hábitats más naturales como los ecosistemas costeros y zonas húmedas y riparias. De hecho, tanto en Cerdeña como en las Baleares, los hábitats más susceptibles a las invasiones biológicas son principalmente los costeros y los humedales.

Una mayor invasibilidad de los ecosistemas costeros es consecuencia de la fuerte vocación turística de ambos territorios y del Mediterráneo en general, que propicia la degradación de estos hábitats, así como el desarrollo en éstos de actividades como la jardinería que actúan como vectores eficientes en la introducción de especies exóticas (Sanz Elorza \& al., 2009). Por otra parte, muchos de estos táxones se ven favorecidos porque vienen de regiones con clima mediterráneo, como A. saligna, procedente de Australia o Carpobrotus sp. pl., de la región Capense, o por pertenecer a familias de plantas suculentas, como Aizoaceae, Cactaceae y Agavaceae, acostumbradas a las altas temperaturas, largos períodos de sequía y la ausencia de heladas importantes en invierno.

Incluso la invasión de los humedales y las zonas ribereñas está estrechamente vinculada a la degradación de los hábitats debido a actividades humanas, siempre mayores en las cercanías de zonas residenciales y en tierras bajas (Schnitzler \& al., 2007; Bacchetta \& al., 2008; Sartori \& Assini, 2008; Blondel \& Médail, 2009). Además, los ambientes acuáticos presentan una especial sensibilidad a los procesos de invasión debido, entre otras causas, a la facilidad de dispersión de los propágulos y semillas por este medio así como al elevado dinamismo que presenta su vegetación (Cronk \& Siobhan Fennessy, 2001). Esto sucede especialmente en los hábitats más frágiles donde la resiliencia y capacidad de recuperación de la naturalidad es menor, y en Cerdeña esto ocurre en particular en las zonas húmedas y riparias. En este sentido, conviene señalar que las especies exóticas que colonizan las zonas húmedas son consideradas peligrosas, independientemente de su potencial invasor en otras regiones con clima mediterráneo (Brunel \& Tison, 2005), además de estar incluidas en el Global Compendium of Weeds (Randall, 2002). 
En cambio, los hábitats naturales más maduros y estables, como los bosques y hábitats arbustivos, se revelan menos sujetos a la incorporación de flora exótica. En particular los bosques se muestran especialmente resistentes a las invasiones. Si bien no hay especies invasoras de este último hábitat, la presencia de Opuntia ficus-indica (L.) Mill. y O. subulata (Muehlenpf.) Engelm. en zonas de maquia degradada pone de relieve la sensibilidad de los hábitats arbustivos a las invasiones en presencia de perturbaciones.

$\mathrm{El}$ análisis de los arqueófitos y neófitos en los diferentes hábitats en Cerdeña y las Islas Baleares pone de manifiesto la diferencia de uso en el tiempo de las especies introducidas antes y después de 1500. Los arqueófitos son especies cuya introducción se ha producido desde tiempos antiguos, que a menudo se consideran parte integrante de la flora local y no requieren control o erradicación si no fuera por unos pocos casos de arqueófitos invasores (p. ej., A. donax, R. comunis). Su presencia es evidente sobre todo en las zonas donde la agricultura tradicional todavía persiste, en los bordes de los campos o en las antiguas tierras de cultivo. Por su parte, como ya han puesto de manifiesto algunos autores (Fraga \& García, 2004), los neófitos son táxones introducidos principalmente para usos ornamentales (Reichard \& White, 2001; Mack, 2003; Sanz Elorza \& al., 2009) que se han escapado de jardines o parques, llegando a naturalizarse en los hábitats más antropizados, como los sinantrópicos, una tendencia común a otros territorios (Panetta, 1993; Mack \& Erneberg, 2002; Pyšek \& al., 2002; DehnenSchmutz \& al., 2007a, 2007b; Milbau \& Stout, 2008).

\section{Agradecimientos}

El presente trabajo ha sido financiado en parte por la Escuela de Doctorado en Ingeniería y Ciencias para el Medio Ambiente y el Territorio de la Universidad de Cagliari y ha recibido el apoyo de la RAS.

Los autores desean dar las gracias por su cooperación a Alessia Nucci, Elisabetta Pirodda, Roberto Pisu y M. ${ }^{a}$ Eugènia Rodrigo Santamalia. Asimismo desean agradecer la revisión crítica de los textos llevada a cabo por Anna Traveset y los dos revisores anónimos de esta publicación.

\section{Referencias bibliográficas}

Álvarez,W. 1972. Rotation of Corsica-Sardinia microplate. Nature 235: 103-105.

Bacchetta, G. \& Pontecorvo, C. 2005. Contribution to the knowledge of the endemic vascular flora of Iglesiente (SW SardiniaItaly). Candollea 60: 481-501.

Bacchetta, G., Bagella, S., Biondi, E., Farris, E., Filigheddu, R. \& Mossa, L. 2009a. Vegetazione forestale e serie di vegetazione della Sardegna (con rappresentazione cartografica alla scala 1:350.000). Fitosociologia suppl. 1, 46:3-82.
Bacchetta, G., Dettori, C.A., Mascia, F., Meloni, F. \& Podda, L. 2010. Assessing the potential invasiveness of Cortaderia selloana in Sardinian wetlands through seed germination study. Plant Biosystems 144, 3: 518-527.

Bacchetta, G., Mascia, F., Mayoral García-Berlanga, O. \& Podda, L. 2008. Dati preliminari sulla flora aliena delle aree umide della Sardegna (Italia). Memorie Società Italiana Scienze Naturali e Museo Civico Storia Naturale di Milano 36: 41.

Bacchetta, G., Mayoral García-Berlanga, O. \& Podda, L. 2009b. Catálogo de la flora exótica de Cerdeña (Italia). Flora Montiberica 41,1:35-61.

Bazos, I., Kokkoris, Y., Zikos, A., Andriopoulos, P., Delipetrou, P., Georghiou, K., Yannitsaros, A. \& Arianoutsou, M. 2009. The alien vascular flora of Greece: Floristic analysis and chorology. Bocconea 23: 281-284.

Blasi, C., Pretto, F. \& Celesti-Grapow, L. 2008. La watch-list della flora alloctona d'Italia. Memorie Società Italiana Scienze Naturali e Museo Civico Storia Naturale di Milano 36: 7-8.

Blondel, J. \& Médail, F. 2009. Biodiversity and conservation. In: Woodward, J.C. (ed.), The Physical Geography of the Mediterranean: 615-650. Oxford University Press. Oxford.

Bolòs, O., Molinier, R. \& Montserrat, P. 1970. Observations phytosociologiques dans l'île de Minorque. Acta Geobotanica Barcinonensia 5: 1-150.

Brundu, G., Camarda, I., Hulme, P.E., Vilà, M., Troumbis, A., Traveset, A., Moragues, E. \& Suehs, C. 2004. Comparative analysis of the abundance and distribution of alien plants on Mediterranean islands. Proceedings 10th MEDECOS, International Conference Ecology Conservation and Management of Mediterranean Climate Ecosystems: 1-9. Rhodes. Greece.

Brundu, G., Camarda, I., Hulme, P.E., Vilà, M., Troumbis, A., Traveset, A., Moragues, E. \& Suhes, C.M. 2004. Comparative analysis of the abundance and distribution of alien plants on Mediterranean islands. In: Arianoutsou, M. \& Papanastasis, V. (eds.), Ecology, Conservation and Management of Mediterranean Climate Ecosystems. Proceedings 10th MEDECOS Conference, 25 April - 1 May 2004, Rhodes, Greece: 1-9. Millipress. Rotterdam.

Brunel, S. \& Tison, J.M. 2005. A method of selection and hierarchization of the invasive and potentially invasive plants in continental Mediterranean. In: Brunel, S. (ed.), Invasive plants in Mediterranean type regions of the world. Proceedings, Mèze (France), 25-27 May 2005: 49-63. Environmental Encounters Series 59. Council of Europe Publishing. Strasbourg.

Celesti-Grapow, L., Pretto, F., Brundu, G., Carli, E. \& Blasi, C. (eds.) 2009a. Plant invasion in Italy. An overview. Palombi \& Partner. Rome.

Celesti-Grapow, L., Alessandrini, A., Arrigoni, P.V., Banfi, E., Bernardo, L., Bovio, M., Brundu, G., Cagiotti, M.R., Camarda, I., Carli, E., Conti, F., Fascetti, S., Galasso, G., Gubellini, L., La Valva, V., Lucchese, F., Marchiori, S., Mazzola, P., Peccenini, S., Pretto, F., Poldini, L., Prosser, F., Siniscalco, C., Villani, M.C., Viegi, L., Wilhalm, T. \& Blasi, C. 2009b. The inventory of the non-native flora of Italy. Plant Biosystems 143: 1-45.

Clout, M.N. \& Veitch, C.R. 2002. Turning the tide of biological invasion: the potential for eradicating invasive species. In: Clout, M.N. \& Veitch, C.R. (eds.), Turning the tide: the Eradicatiof Invasive Species: 1-3. IUCN Species Specialist Group. Gland. Switzerland and Cambridge. United Kingdom.

Comunitat Valenciana, 2009. Decreto 213/2009, de 20 de noviembre de 2009, del Consell, por el que se aprueban medidas para el control de especies exóticas invasoras en la Comunitat Valenciana. Diari Oficial de la Comunitat Valenciana, 24 de novembre de 2009, num. 6151: 42326-42332. 
Conti, F., Abbate, G., Alessandrini, A. \& Blasi, C. (eds.) 2005. An annotated Checklist of the Italian Vascular Flora. Palombi Editori. Roma.

Conti, F., Alessandrini, A., Bacchetta, G., Banfi, E., Barberis, G., Bartolucci, F., Bernardo, L., Bonacquisti, S., Bouvet, D., Bovio, M., Brusa, G., Del Guacchio, E., Foggi, B., Frattini, S., Galasso, G., Gallo, L., Gangale, C., Gottschlich, G., Grünanger, P., Gubellini, L., Iiriti, G., Lucarini, D., Marchetti, D., Moraldo, B., Peruzzi, L., Poldini, L., Prosser, F., Raffaelli, M., Santangelo, A., Scassellati, E., Scortegagna, S., Selvi, F., Soldano, A., Tinti, D., Ubaldi, D., Uzunov, D. \& Vidali, M. 2007. Integrazioni alla checklist della flora vascolare italiana. Natura Vicentina 10: 5-74.

Cox, G.W. 1999. Alien species in North America and Hawaii: impacts on natural ecosystems. Island Press. Washington DC.

Crawley, M. J., Harvey, P.H. \& Purvis, A. 1996 Comparative ecology of the native and alien floras of the British Isles. Philosophical Transactions of the Royal Society of London, B 351: 12511259.

Cronk, J.K. \& Siobhan Fennessy M. 2001. Wetland plant biology and ecology. Lewis Publishers. New York.

Cronk, Q.C.B. \& Fuller, J.L. 1995. Plant Invaders. Chapman \& Hall. London.

DAISIE. 2009. The Handbook of Alien Species in Europe. Invading Nature: Springer Series in Invasion Ecology. Springer. Amsterdam.

DAISIE. [2010]. DAISIE European Invasive Alien Species Gateway [http://www.europe-aliens.org].

D’Antonio, C.M., Chambers, J.C., Loh, R. \& Tunison, J.T. 2009. Applyng Ecological Concepts to the Management of Widespread Grass Invasions. In: Inderjit (eds.), Management of Invasive Weeds 5: 123-149. Invading Nature: Springer Series in Invasion Ecology. Springer. New York

Daehler, C.C., Denslow, J.S., Ansari, S. \& Kuo, H.-C. 2004. A risk assessment system for screening out invasive pest plants from Hawaii and other Pacific Islands. Conservation biology 18: 360-368.

Dal Cin D’Agata, C., Skoula, M. \& Brundu, G. 2009. A preliminary inventory of the alien flora of Crete (Greece). Bocconea 23: 301-315.

Debussche, M., Lepart, J. \& Dervieux, A. 1999. Mediterranean land-scape changes: evidence from old postcards. Global Ecology and Biogeography 8: 3-15.

Dehnen-Schmutz, K., Touza, J., Perrings, C. \& Williamson, M. 2007a. A century of the ornamental plant trade and its impact on invasion success. Diversity and Distributions 13: 527-534.

Dehnen-Schmutz, K., Touza, J., Perrings, C. \& Williamson, M. 2007b. The horticultural trade and ornamental plant invasions in Britain. Conservation Biology 21: 224-231.

Denslow, J.S. 2003. Weeds in paradise: thoughts on the invasibility of tropical islands. Annals of the Missouri Botanical Garden 90: 119-127.

Di Castri, F. 1991. An ecological overview of the five regions of the world with Mediterranean climate. In: Groves, R.H. \& Di Castri, F. (eds.), Biogeography of Mediterranean invasions: 3-15. Cambridge University Press. Cambridge.

Fraga i Arguimbau, P., Mascaró Sintes, C., Carreras Martí, D., García Febrero, O., Pallicer Allés, X., Pons Gomila, M., Seoane Barber, M. \& Truyol Olives, M. 2004. Catàleg de la flora vascular de Menorca. Institut Menorquí d'Estudis. Maó.

Fraga, P. \& García, O. 2004. Notes i contribucions al coneixement de la flora de Menorca (VI). Bolletí de la Societat d'Història Natural de les Balears 47: 143-152.
Fraga, P., Mascaró, C., Carreras, D., García, O., Pons, M. \& Truyol, M. 2001. Notes i contribucions al coneixement de la flora de Menorca (II). Bolletí de la Societat d'Història Natural de les Balears 44: 73-79.

Fraga, P., Mascaró, C., Carreras, D., García, O., Pons, M. \& Truyol, M. 2002. Notes i contribucions al coneixement de la flora de Menorca (III). Bolletí de la Societat d'Història Natural de les Balears 45: 69-79.

Fraga, P., Mascaró, C., García, O., Pallicer, X., Pons, M. \& Truyol, M. 2000. Notes i contribucions al coneixement de la flora de Menorca. Bolletí de la Societat d'Història Natural de les Balears 43: 63-75.

Fraga, P. \& Pallicer, X. 1998. Notes florístiques de Menorca. Bulletí de la Institució Catalana d'Història Natural (Barcelona) 66: 35-40.

Fritts, T.H. \& Rodda, G.H. 1998. The role of introduced species in the degradation of island ecosystems: a case history of Guam. Annual Review of Ecology and Systematics 29: 113-140.

García-Berthou, E., Ballesteros, E. \& Sanz-Elorza, M. 2008. Múltiples orígines y vías de introducción de las especies invasoras. In: Vilà, M. \& al. (eds), Invasiones Biológicas: 29-40. Consejo Superior de Investigaciones Científicas-Divulgación. Madrid.

Giardina, G., Raimondo, F.M. \& Spadaro, V. 2007. A catologue of plants growing in Sicily. Bocconea 20: 5-582.

Gillespie, T.W., Chu, J. \& Pau, S. 2008. Non-Native Plant Invasion of the Hawaiian Islands. Geography Compass 2: 1241-1265.

Heywood, V.H. 1989. Patterns, extens and modes of invasions of terrestrial plants. In: Drake, J.A. \& al. 1989 (eds.), Biological invasions, a global perspective. John Wiley and Sons, Chichester. Reino Unido.

Hernández, G., Lahmann, E. \& Pérez-Gil, R. 2002. Invasores en Mesoamérica y el Caribe: Invasives in Mesoamerica and the Caribbean. IUCN. San Jose. Costa Rica.

Hill, M.O., Roy, D.B. \& Thompson, K. 2002. Hemeroby, urbanity and ruderality: bioindicators of disturbance and human impact. Journal of Applied Ecology 39: 708-720.

Hulme, P.E. 2006. Beyond control: wider implications for the management of biological invasions. Journal of Applied Ecology 43: 835-847.

Hulme, P.E. 2007. Biological invasion in Europe: drivers, pressures, states, impact and responses. In: Hester, R. \& Harrison, R.M. (eds.), Biodiversity under threat issues in enviromental science and technology 25: 56-80. Royal Society of Chemistry. Cambridge.

Hulme, P.E., Bacher, S., Kenis, M., Klotz, S., Kühn, I., Minchin, D., Nentwig, W., Olenin, S., Panov, V., Pergl, J., Pyšek, P., Roque, A., Sol, D., Solarz, W. \& Vilà, M. 2008a. Grasping at the routes of biological invasions: a framework for integrating pathways into policy. Journal of Applied Ecology 45: 403 414.

Hulme, P.E., Brundu, G., Camarda, I., Dalias, P., Lambdon, P., Lloret, F., Médail, F., Moragues, E., Suehs, C., Traveset, A., Troumbis, A. \& Vilà, M. 2008b. Assessing the risks to Mediterranean islands ecosystems from alien plant introductions. In: Tokarska-Guzik, B. \& al. (eds.), Plant invasions: Human perception, ecological impacts and management: 39-56. Backhuys Publishers. Leiden.

Jeanmonod, D. 1998. Les plantes introduites en Corse: impact, menaces et propositions de protection de la flore indigène. Biocosme Mésogéen 15: 45-68.

Kitayama, K. \& Mueller-Dombois, D. 1995. Biological invasion on an oceanic island mountain: Do alien plant species have wider ecological ranges than native species? Journal of vegetation $S_{c i}$ ence 6: 667-674. 
Kotanen, P.M. 2004. Revegetation following soil disturbance and invasion in a californian meadow: a 10-year history of recovery. Biological Invasions 6: 245-254.

Kueffer, C. 2006. Impacts of woody invasive species on tropical forests of the Seychelles. Diss. ETH No. 16602. Department of Environmental Sciences. ETH Zurich. Zurich.

Kueffer, C., Daehler, C.C., Torres-Santana, C.W., Lavergne, C., Meyer, J.-Y., Otto, R. \& Silva, L. 2010. A global comparison of plant invasions on oceanic islands. Perspectives in Plant Ecology, Evolution and Systematics 12, 2: 145-161.

Kunkel, G. 1993. Die Kanarischen Inseln und ibre Pflanzenwuelt. 3 Aufl. Stuttgart. Alemania.

Ladero Álvarez, M., Díaz González, T.E., Penas Merino, A., RivasMartínez, S. \& Valle Gutiérrez, C. 1987. Datos sobre la vegetación de las Cordilleras Central y Cantábrica. Itinera Geobotanica 1:3-147.

Lambdon, P.W., Pyšek, P., Basnou, C., Hejda, M., Arianoutsou, M., Essl, F., Jarošík, V., Pergl, J., Winter, M., Anastasiu, P., Andriopoulos, P. Bazos, I., Brundu, G., Celesti-Grapow, L., Chassot, P., Delipetrou, P., Josefsson, M., Kark, S., Kokkoris, Y., Marchante, H., Perglová, I., Pino, J., Vilà, M., Zikos, A., Roy, D. \& Hulme, P.E., 2008. Alien flora of Europe: species diversity, temporal trends, geographical patterns and research needs. Prestia 80: 101-149.

Le Houérou, H.N. 1991. Plant invasions in the rangelands of the isoclimatic mediterranean zone. In: Groves, R.H. \& Di Castri, F. (eds.), Biogeography of Mediterranean invasions: 393-404. Cambridge University Press. Cambridge.

Le Neindre, M. 2002. Les espèces introduites et envahissantes dans les îles Méditerranéennes: etat dess lieux et propositions d'action. Université de Corse, Faculté des Sciences et Techniques.

Levine, J.M., Adler, P.B. \& Yelenik, S.G. 2004. A meta-analysis of biotic resistance to exotic plant invasions. Ecology Letters 7: 975-989.

Lloret, F., Médail, F., Brundu, G., Mus, M. \& Hulme, P.E. 2004. Local and regional abundance of exotic plant species on Mediterranean islands: are species traits important? Global Ecology and Biogeography 13: 37-45.

Lloret, F., Médail, F., Brundu, G., Camarda, I., Moragues, E., Rita, J., Lambdon, P. \& Hulme, P.E. 2005. Species attributes and invasion success by alien plants on Mediterranean islands. Journal of Ecology 93: 512-520.

Lockwood, J.L., Cassey, P. \& Blackburn, T. 2005. The role of propagule pressure in explaining species invasions. Trends in Ecology and Evolution 20: 223-228.

Lodge, D.M. 1993. Biological invasions: lessons for ecology. Trends in Ecology and Evolution 8: 133-137.

Lonsdale, M.W. 1999. Global patterns of plant invasion and the concept of invasibility. Ecology 80: 1522-1536.

Loope, L.L. \& Mueller-Dombois, D. 1989. Characteristics of invaded islands, with special reference to Hawaii. In: Drake, J.A. $\&$ al. (eds.), Biological invasions: A global perspective, SCOPE 37: 257-280. John Wiley and Sons. New York.

Mack, R.N. 2000. Cultivation fosters plant naturalization by reducing environmental stochasticity. Biological Invasions 2: 111-122.

Mack, R.N. 2003. Global plant dispersal, naturalization, and invasion: pathways, modes, and circumstances. In: Ruiz, G.M. \& Carlton, J.T. (eds.), Invasive species: vectors and management strategies: 3-30. Island Press. Washington.

Mack, R.N. \& Erneberg, M. 2002. The United States naturalized flora: largely the product of deliberate introductions. Annals of the Missouri Botanical Garden 89: 176-189.
Mack, R., Simberloff, D., Lonsdale, W., Evans, H., Clout, M. \& Bazzaz, F. 2000. Biotic invasions. Causes, epidemiology, global consequences and control. Ecological Applications 10: 689-710.

Madon, O. \& Médail, F. 1997. The ecological significance of annuals on a Mediterranean grassland (Mt Ventoux, France). Plant Ecology 129: 189-199.

Mansion, G., Rosenbaum, G., Schönenberger, N., Bacchetta, G., Rosselló, J.A. \& Conti, E. 2008. Phylogenetic analysis informed by geological history supports multiple, sequential invasions of the mediterranean basin by the angiosperm family Araceae. Systematic Biology 57: 269-285.

Mayer, A. 1995. Comparative study of the coastal vegetation of Sardinia (Italy) and Crete (Greece) with respect to the effects of human influence. Libri botanici 15. IHW-Verlag. Eching.

Milbau, A. \& Stout, J.C. 2008. Factors Associated with Alien Plants Transitioning from Casual, to Naturalized, to Invasive. Conservation Biology 22: 308-317.

Miller, C., Kettunen, M. \& Shine, C. 2006. Scope options for EU action on invasive alien species (IAS). Final report for the European Commission. Institute for European Environmental Policy (IEEP). Brussels. Belgium.

Mooney, H.A. \& Hobbs, R.J. 2000. Invasive species in a changing world. Island Press. Washington DC.

Moragues Botey, E. \& Rita Larrucea, J. 2005. Els vegetals introduits a les Illes Balears. Documents tècnics de conservaciò, IIa època, núm. 11. Govern de les Illes Balears. Conselleria de Medi Ambient. Palma.

Mueller-Dombois, D. 1981. Island ecosystems: what is unique about their ecology? In: Mueller-Dombois, D. \& al. (eds.), Island ecosystems: biological organisation in selected Hawaiian communities: 485-501. Hutchinson Ross Publishing Company. Woods Hole, Massachusetts.

Natali, A. \& Jeanmonod, D. 1996. Flore analytique des plantes introduites en Corse. In: Jeanmonod, D. \& Burdet, H.M. (eds.), Compléments au Prodrome de la Flore Corse. Annexe n. 4. Conservatoire et Jardin Botaniques, Genève.

O’Dowd, D.J., Green, P.T. \& Lake, P.S. 2003. Invasional meltdown on an oceanic island Ecology Letters 6: 812-817.

Olesen, J.M., Eskildsen, L.I. \& Venkatasamy, S. 2002. Invasion of pollination networks on oceanic islands: importance of invader complexes and endemic super generalists. Diversity and Distributions 8: 181-192.

Pauchard, A., Cavieres, L.A. \& Bustamante, R.O. 2004. Comparing alien plant invasions among regions with similar climates: where to from here? Diversity and Distributions 10: 371-375.

Panetta, F.D. 1993. A system of assessing proposed plant introduction for weed potential. Plant Protection Quarterly 8: 10-14.

Pignatti, S. 1982. Flora d'Italia 1-3. Edagricole. Bologna.

Pla, V., Sastre, B. \& Llorens, Ll. 1992. Aproximació al catàleg de la flora de les Illes Balears. Universitat de les Illes Balears. Palma.

Pyšek, P. \& Richardson, D.M. 2006. The biogeography of naturalization in alien plants. Journal of Biogeography 12: 2040-2050.

Pyšek, P., Lambdon, P.W., Arianoutsou, M., Kühn, I., Pino, J. \& Winter, M. 2009. Alien Vascular Plants of Europe. In: DAISIE. 2009, The Handbook of Alien Species in Europe: 43-61. Invading Nature. Springer Series in Invasion Ecology. Springer. Amsterdam.

Pyšek, P., Richardson, D.M., Rejmánek, M., Webster, G.L., Williamson, M. \& Kirschner, J. 2004. Alien plants in checklist and floras: towards better communication between taxonomist and ecologists. Taxon 53: 131-143. 
Pyšek, P., Sadlo, J. \& Mandak, B. 2002. Catalogue of alien plants of the Czech Republic. Preslia 74: 97-186.

Randall, R.P. 2002. A global compendium of weeds. RG \& FJ Richardson Puplisher. Australia.

Randall, J.M., Morse, L.E., Benton, N., Hiebert, R., Lu, S. \& Killefer, T. 2008. The invasive species assessment protocol: a tool for creating regional and national lists of invasive nonnative plants that negatively impact biodiversity. Invasive Plant Science and Management 1:36-49.

Raunkiaer, C. 1934. The life form of plants and statistical plant geography. Oxford University Press. Oxford.

Reaser, J.K., Meyerson, L.A., Cronk, K., De Poorter, M., Eldrege, L.G., Green, E., Kairo, M., Latasi, P., Mack, R.N., Mauremootoo, G., O’Dowd, D., Orapa, W., Sastroutomo, S., Saunders, A., Shine, C., Thrainsson, S. \& Vaiutu, L. 2007. Ecologi$\mathrm{cal}$ and socioeconomic impacts of invasive alien species in island ecosystems. Enviromental Conservation 34: 98-111.

Reichard, S. H., \& White, P. 2001. Horticulture as a pathway of invasive plant introductions in the United States. BioScience 51: 103-113.

Rejmánek, M., Richardson, D.M. \& Pyšek, P. 2004. Plant invasion and invasibility of plant communities. In: Van Der Maarel (eds.), Vegetation ecology: 332-355. Blackwell Publishing. Malden.

Ricciardi, A. \& Cohen, J. 2007. The invasiveness of an introduced species does not predict its impact. Biological Invasions 9: <

309-315.

Richardson, D.M. \& Pyšek, P. 2006. Plant invasions: merging the concepts of species invasiveness and community invasibility. Progress in Physical Geography 30: 409-431.

Richardson, D.M., Pyšek, P., Rejmánek, M., Barbour, M.G., Panetta, F.D. \& West, C.J. 2000. Naturalization and invasion of alien plants: concepts and definitions. Diversity and Distributions 6: 93-107.

Rita Larrucea, J. \& Payeras Coll, T. 2006. Biodiversidad de las plantas vasculares de las Islas Baleares. Orsis 21: 41-58.

Rivas-Martínez, S. 2007. Mapa de series, geoseries y geopermaseries de vegetación de España. Itinera Geobotanica (nueva serie) 17:5-436.

Rodríguez, J.J. 1904. Flórula de Menorca. Imp. Fábregas. Maó.

Rosenbaum, G., Lister, G. S. \& Duboz, C. 2002. Reconstruction of the tectonic evolution of the western Mediterranean since the Oligocene. Journal Virtual Explorer 8: 107-130.

Sanz Elorza, M., Dana Sánchez, E.D. \& Sobrino Vesperinas, E. 2004. Atlas de las plantas alóctonas invasoras en España. Ministerio de Medio Ambiente. Madrid.

Sanz Elorza, M., Mateo, R.G. \& González Bernardo, F. 2009. The historical role of agricultura and gardening in the introduction of alien plants in the western Mediterranean. Plant Ecology 202: 247-256.

Sartori, F. \& Assini, S. 2008. Osservazioni su specie vegetali esotiche invasive lungo i fiumi padani. Memorie Società Italiana Scienze Naturali e Museo Civico Storia Naturale di Milano 36: 15.

Schippers, P., Van Groenendael, J.M., Vleeshouwers, L.M. \& Hunt, R. 2001. Herbaceous plant strategies in disturbed habitats. Oikos 95: 198-210.

Schnitzler, A. Hale, B.W. \& Alsum, E.M. 2007. Examining native and exotic species diversity in European riparian forests. Biological Conservation 138: 146-156.
Sherley, G. 2000 (eds.). Invasive species in the Pacific: a technical review and draft regional strategy. South Pacific Regional Enviroment Programme. Apia. Samoa.

Simberloff, D. 1995. Why do introduced species appear to devastate islands more than mainland areas? Pacific Science 49: 87-97.

Simberloff, D. 2000. Extinction-proponess of island species: causes and management implications. Raffles Bulletin of Zoology 48: $1-9$.

Speranza, F., Villa, I.M., Sagnotti, L., Florindo, F., Cosentino, D., Cipollari, P. \& Mattei, M. 2002. Age of the Corsica-Sardinia rotation and Liguro-Provencal Basin spreading: New paleomagnetic and Ar/Ar evidence. Tectonophysics 347: 231-251.

Suehs, C.M., Affre, L. \& Médail, F. 2004. Invasion dynamics of two alien Carpobrotus (Aizoaceae) taxa on a Mediterranean island: I. Genetic diversity and introgression. Heredity 92:31-40.

Suehs, C.M., Médail, F. \& Affre, L. 2001. Ecological and genetic features of the invasion by the alien Carpobrotus plants in Mediterranean island habitats. In: Brundu, G. \& al. (eds.), Plant invasions: species ecology and ecosystem management: 145-158. Backhuys Publishers. Leiden.

Tassin, J., Lavergne, C., Muller, S., Blanfort, V., Baret, S., Le Bourgeois, T., Triolo, J. \& Riviere, J.-N. 2006. Bilan des connaissances sur les consequences ecologiques des invasions de plants a l'ile de La Reunion (Archipel des Mascareignes, Ocean Indien). Revue d'Ecologie (la Terre et la Vie) 61:35-52.

Towns, D.R. \& Ballantine, W.J. 1993. Conservation and restauration of New-Zealand islands ecosystems. Trends in Ecology and Evolution 8: 452-457.

Vilà, M. \& Muñoz, I. 1999. Patterns and correlates of exotic and endemic plant taxa in the Balearic islands. Ecologia Mediterránea 25: 153-161.

Vilà, M., Meggaro, Y. \& Weber, E. 1999. Preliminary analysis of the naturalized flora of northern Africa. Orsis 14: 9-20.

Vilà, M., Tessier, M., Gimeno, I., Moragues, E., Traveset, A., De La Bandera, M.C., Suehs, C.M., Médail, F., Affre, L., Galanidis, A., Dalias, P., Petsikos, B., Carta, L., Manca, M. \& Brundu, G. 2004. In: Arianoutsou, M. \& Papanastasis, V. (eds.), Ecology, conservation and management of Mediterranean climate ecosystems. Proceedings 10th MEDECOS Conference, 25 April 1 May 2004, Rhodes, Greece: 1-9. Millipress. Rotterdam.

Vilà, M., Tessier, M., Suehs, C.M., Brundu, G., Carta, L., Galanidis, A., Lambdon, P., Manca, M., Médail, F., Moragues, E., Traveset, A., Troumbis, A.Y. \& Hulme, P.E. 2006. Local and regional assessment of the impacts of plant invaders on vegetation structure and soil properties of Mediterranean islands. Journal of Biogeography 33: 853-861.

Vitousek, P.M. 1987. Biological invasion by Myrica faya alters ecosystems in Hawaii. Science 238: 802-804.

Weber, E. 2003. Invasive plant species of the world. A reference guide to environmental weeds. CABI publishing. Wallingford.

Wittenberg, R. \& Cock, M.J.W. 2001. Invasive alien species: a toolkit for best prevention and management practices. CAB International. Wallingford.

Editor asociado: A. Traveset Recibido: 3-III-2010 Aceptado: 20-VII-2010 